\title{
mTOR signalling and cellular metabolism are mutual determinants in cancer
}

\author{
Dirk Mossmann, Sujin Park and Michael N. Hall* \\ Biozentrum, University of Basel, Basel, Switzerland. \\ *e-mail: m.hall@unibas.ch
}

\begin{abstract}
Oncogenic signalling and metabolic alterations are interrelated in cancer cells. mTOR, which is frequently activated in cancer, controls cell growth and metabolism. mTOR signalling regulates amino acid, glucose, nucleotide, fatty acid and lipid metabolism. Conversely, metabolic inputs, such as amino acids, activate mTOR. In this Review, we discuss how mTOR signalling rewires cancer cell metabolism and delineate how changes in metabolism, in turn, sustain mTOR signalling and tumorigenicity. Several drugs are being developed to perturb cancer cell metabolism. However, their efficacy as stand-alone therapies, similar to mTOR inhibitors, is limited. Here, we discuss how the interdependence of mTOR signalling and metabolism can be exploited for cancer therapy.
\end{abstract}

Cancer cell growth is driven by aberrant signalling and metabolic reprogramming ${ }^{1}$. Cancer cells reprogramme their metabolism to ensure survival and proliferation in an often nutrient-scarce and stressful microenvironment. Metabolic alterations affect both catabolic pathways for production of ATP and precursor molecules and anabolic pathways for the synthesis of biomass. Many cancer-specific metabolic alterations have been described, including aberrant metabolism of amino acids, glucose, nucleotides, fatty acids and lipids. Metabolic reprogramming is often mediated by oncogenic signalling. In particular, mTOR signalling is commonly activated in tumours and controls cancer cell metabolism by altering expression and/or activity of a number of key metabolic enzymes ${ }^{2,3}$. Conversely, metabolic alterations, such as increased glucose or amino acid uptake, impact mTOR signalling. Thus, an integrated understanding of the crosstalk between mTOR signalling and cancer metabolism can aid in the development of novel therapeutic strategies. Here, we review recent findings on mTOR signalling, with a focus on cancer-specific metabolic alterations. We suggest that targeting both mTOR signalling and cancer cell-specific metabolic dependencies can be synergistic.

\section{Mechanisms of mTOR activation}

The atypical serine/threonine kinase mTOR is a master regulator of cell growth and metabolism. mTOR promotes anabolic processes such as ribosome biogenesis and protein, nucleotide, fatty acid and lipid synthesis and inhibits catabolic processes such as autophagy ${ }^{2}$. It is part of two structurally and functionally distinct complexes mTOR complex 1 (mTORC1) and mTORC2 (Fig. 1). mTORC1 contains mTOR, regulatoryassociated protein of mTOR (RAPTOR) and mammalian lethal with sec-13 protein 8 (mLST8) and is rapamycin sensitive. mTORC2 contains mTOR, rapamycin-insensitive companion of mTOR (RICTOR), mammalian stress- activated MAPK-interacting protein 1 (mSIN1; also known as MAPKAP1) and $\mathrm{mLST} 8$ and is insensitive to acute 
rapamycin treatment.

\section{Activation of mTORC1 by growth factors and nutrients}

The activation of mTORC1 is dependent on nutrients and growth factors. In response to nutrients, mTORC1 translocates from the cytoplasm to the lysosomal surface, where it is activated by growth factors via PI3K-AKT signalling. Growth factors, for instance, insulin, activate AKT4 via a cognate receptor, phosphoinositide- dependent kinase 1 (PDK1) and PI3K (Fig. 1). AKT inhibits the TSC1-TSC2 complex5, which is a GTPase- activating protein (GAP) for the small GTPase RHEB6. GTP-bound RHEB directly binds and activates mTORC1 at the lysosome ${ }^{7,8}$ (Fig. 1).

\section{Nutrient-induced lysosomal translocation of mTORC1}

Nutrients, in particular amino acids, promote lysosomal localization of mTORC1 via the RAS-related GTP- binding proteins (RAGs) ${ }^{9}$, thereby enabling mTORC1 to encounter RHEB. RAGs are small GTPases that form obligate heterodimers. RAGA or RAGB associates with RAGC or RAGD. In the active state, GTP-bound RAGA or RAGB and GDP-bound RAGC or RAGD bind RAPTOR and thereby recruit mTORC1 to the lysosomal surface. The nucleotide binding status of the RAGs is tightly regulated by amino acids ${ }^{9}$ obtained from intracellular synthesis, protein turnover or extracellular sources via specific transporters (Fig. 1; for details, see following sections). Among the amino acids, leucine, arginine and glutamine are the most effective activators of mTORC1. Leucine and arginine bind to sestrin 2 and CASTOR1, respectively, ultimately to activate the RAGs and mTORC1 (for details, see Fig. 1, ref. ${ }^{10}$ and references therein). The lysosomal amino acid transporter SLC38A9 promotes mTORC1 activation by exporting essential amino acids to the cytoplasm ${ }^{11}$, where, for example, leucine can bind sestrin 2. Leucine export is stimulated by arginine binding to SLC38A9 (ref. ${ }^{11}$ ).

Glutamine activates RAGs by promoting glutaminolysis. During glutaminolysis, glutaminase (GLS) and glutamate dehydrogenase (GDH) convert glutamine to $\alpha$ ketoglutarate $(\alpha \mathrm{KG})$, which ultimately activates mTORC1 via prolyl hydroxylases (PHDs) by promoting GTP loading of RAGB12 (Fig. 1). Leucine also stimulates $\alpha \mathrm{KG}$ production by directly binding and allosterically activating GDH. Furthermore, glutamine activates mTORC1 independently of RAGs via the small GTPase ADPribosylation factor 1 (ARF1) ${ }^{13}$ Amino acids also signal through folliculin (FLCN) and its binding partners FLCN-interacting proteins 1 or 2 (FNIP1 or FNIP2), which act as GAPs for RAGC and/or RAGD.

\section{Activation of mTORC2 by growth factors}

In contrast to mTORC1, growth factor signalling alone is sufficient to activate mTORC2, but the mechanism is still incompletely understood. Growth factors, for example, insulin, promote activation of PI3K and production of phosphatidylinositol$(3,4,5)$-triphosphate (PIP3), which in turn binds mSIN1 to relieve an mSIN1- mediated inhibitory effect on mTORC2 (ref. ${ }^{14}$ ). PI3K also activates mTORC2 by promoting association of mTORC2 with the ribosome ${ }^{15}$. 


\section{mTORC1 and mTORC2 signalling effectors}

mTORC1 and mTORC2 regulate cellular growth and metabolism either through direct phosphorylation of key metabolic enzymes or indirectly through downstream signalling effectors. mTORC1 directly activates ribosomal protein S6 kinase (S6K) and inhibits eIF4E binding protein (4EBP) to increase translation, including translation of metabolic enzymes and metabolism-related transcription factors ${ }^{2}$. In addition, mTORC1 and S6K regulate metabolic enzymes directly (see below). mTORC2 promotes metabolism mainly via activation of AKT2. S6K and AKT not only regulate metabolic enzymes but also activate key metabolic transcription factors, such as MYC, hypoxia-inducible factor $1 \alpha(\mathrm{HIF} 1 \alpha)$ and/or HIF $2 \alpha$, FOXO transcription factors and sterol regulatory element binding protein 1 (SREBP1) 2 .

\section{mTOR drives cancer metabolic reprogramming}

In many cancers, mTOR is often activated by mutations in its upstream regulators. These include gain-of-function mutation of PI3K3,16 and loss-of-function mutation of the tumour suppressor PTEN3. Furthermore, other oncogenic signalling pathways, such as RAS signalling, can activate mTOR signalling ${ }^{17}$. Inhibition of mTOR signalling, in particular, inhibition of mTORC1, has been explored as an anticancer strategy but with limited success. For example, rapamycin analogues (rapalogues) do not fully inhibit phosphorylation of the mTORC1 effector 4EBP and lead to compensatory upregulation of mTORC2-AKT activity ${ }^{18}$. As mentioned above, nutrient sensing plays a major role in activation of mTORC1. Therefore, changes in cancer cell metabolism and in levels of intracellular nutrients could also contribute to sustained mTORC1 activation. Conversely, changes in cancer cell metabolism are driven by mTOR signalling, and metabolic pathways promoted by mTOR signalling include amino acid, glucose, nucleotide, fatty acid and lipid metabolism ${ }^{2}$. This mutual determinism might provide novel vulnerabilities in cancer cells, which could be exploited to improve anticancer therapies.

\section{mTOR and amino acid metabolism Glutamine metabolism}

Glutamine is a non-essential amino acid, and yet, many cancer cells depend on extracellular glutamine for survival ${ }^{19}$. Glutamine serves as a nitrogen and carbon donor for the synthesis of amino acids, lipids and nucleotides, and it is used in cancer cells to replenish the tricarboxylic acid (TCA) cycle by anaplerosis ${ }^{20}$. Glutamine is imported into the cell via transporters such as SLC1A5, SLC38A1, SLC38A2 or SLC38A5 that are upregulated in many cancers ${ }^{21}$ (Fig. 2a). Pharmacological or biochemical inhibition of SLC1A5 reduces growth and mTORC1 activity in gastric ${ }^{22}$, prostate ${ }^{23}$ and triplenegative breast ${ }^{24}$ cancer cells. However, blocking a single glutamine transporter might not necessarily be sufficient to prevent tumour growth, as compensatory upregulation of SLC38A1 occurs upon SLC1A5 knockdown in osteosarcoma and cervical cancer cells ${ }^{25}$.

Both mTORC1 and mTORC2 signalling promote glutaminolysis through various mechanisms (Fig. 2a). mTORC1, via S6K, increases translation of $M^{26}{ }^{26}$, which in turn transcriptionally represses miR-23a and miR-23b27. miR-23a and miR-23b post- 
transcriptionally repress GLS ${ }^{27}$. Thus, mTORC1 positively regulates GLS expression to promote glutaminolysis. mTORC1 also promotes glutaminolysis by preventing sirtuin 4 (SIRT4)-mediated GDH inhibition ${ }^{28}$ (Fig. 2a). mTORC2 might also favour glutaminolysis, in this case by inhibiting expression of glutamine synthetase (GS). Mechanistically, the mTORC2 effector AKT prevents nuclear localization of the transcription factors FOXO3 and FOXO4 to inhibit FOXO3-dependent or FOXO4dependent GS expression ${ }^{29}$.

Interestingly, there is evidence that mTOR and glutaminolysis inhibitors have synergistic effects in inhibiting cancer cell growth. In a mouse model for lung squamous cell carcinoma (LSCC), chronic dual mTORC1 and mTORC2 inhibition (hereafter referred to as mTOR inhibition) blocks glycolysis. Chronic mTOR inhibition also provokes an adaptive increase in glutaminolysis that allows tumour cells to circumvent the loss of glycolysis and that results in resistance to mTOR inhibition ${ }^{30}$.

Mechanistically, chronic mTOR inhibition in cancer cells leads to adaptive activation of AKT. This in turn inhibits glycogen synthase kinase $3 \beta$ (GSK3 $\beta$ ), thereby preventing the degradation of the transcription factors JUN and MYC and allowing GLS expression and glutaminolysis (Fig. 2a). Accordingly, resistance to mTOR inhibition could be overcome by addition of the allosteric GLS inhibitor CB-839 $\left(\right.$ ref. $\left.^{30}\right)$, which effectively reduces growth of patient-derived LSCC xenograft ${ }^{30}$.

With the aim of exploiting the dependence of cancer cells on glutamine metabolism, several inhibitors of glutamine uptake and/or catabolism are now being evaluated in preclinical and early-phase clinical trials. For example, a recently developed SLC1A5 inhibitor efficiently reduces mTOR signalling and growth of lung, breast and colorectal cancer cells in vitro and of colorectal xenograft models in vivo ${ }^{31}$. The GLS inhibitor CB-839 is now in clinical trials for several solid tumours and haematological malignancies $^{32,33}$ (Table 1). One ongoing clinical trial is currently evaluating CB-839 in combination with the mTORC1 inhibitor everolimus as a therapy for renal cell carcinoma $(\mathrm{RCC})^{33}$ (Table 1).

\section{Arginine metabolism}

Arginine is a conditionally essential amino acid. Besides being used in protein synthesis, it serves as a precursor for polyamines, nitric oxide, creatine, proline and glutamate. Arginine is taken up into the cell mainly via SLC7A1. Increased expression of the transporter SLC7A1, which increases arginine uptake, has been reported in colorectal cancer ${ }^{34}$, breast cancer cell lines ${ }^{35}$ and hepatocellular carcinoma (HCC) lacking miR-122 expression, a negative regulator of SLC7A1 resistance.

Synthesis of arginine and targeting potential. Arginine is produced by the urea cycle during the conversion of ammonia and aspartate into urea ${ }^{38}$. Carbamoyl phosphate synthetase 1 (CPS1) is located in the mitochondria and produces carbamoyl phosphate from ammonia and bicarbonate as the first step of the urea cycle. Together with ornithine, carbamoyl phosphate is used to generate citrulline. The rate-limiting enzyme in arginine synthesis is argininosuccinate synthetase 1 (ASS1), which forms argininosuccinate from aspartate and citrulline. Argininosuccinate lyase (ASL) then cleaves argininosuccinate into arginine and fumarate ${ }^{38}$ (Fig. 2b). 
ASS1 expression is decreased or even abolished in many cancers including melanoma, RCC, HCC, glioblastoma (GBM), small-cell lung cancer (SCLC), pancreatic cancer and others $^{39,40}$. Interestingly, ASS1 knockdown results in increased mTORC1 activity in osteosarcoma cells, potentially owing to increased aspartate levels ${ }^{41}$. Because ASS1deficient tumours rely on extracellular arginine for their survival, arginine deprivation is a therapeutic strategy for these cancers ${ }^{42}$. Several recombinant arginine-degrading enzymes have been developed and are now being evaluated in clinical trials ${ }^{43-47}$ (Table 1). For example, recombinant human arginase (rhARG) cleaves arginine into urea and ornithine, and pegylated arginine deiminase (ADI-PEG20) degrades arginine by deaminating it to produce citrulline and ammonium. Treatment with rhARG reduces mTORC1 activity and induces cytotoxicity and apoptosis in non- SCLC (NSCLC) cells $^{48}$. However, resistance to these drugs has already been observed. Cancer cells reexpress ASS1 to avoid reliance on extracellular arginine ${ }^{49}$. In the case of ADI-PEG20, treatment activates RAS signalling and subsequently PI3K-AKT signalling, which leads to stabilization of MYC. MYC in turn induces ASS1 expression by competing with HIF1 $\alpha$ for ASS1 promoter binding sites ${ }^{42}$. Therefore, combining recombinant arginine-degrading enzymes with mTOR inhibitors might be beneficial in ASS1negative cancer types. Indeed, co-therapy of ADI-PEG20 with a PI3K inhibitor is more effective than either drug alone in preventing tumour growth in a mouse xenograft model $^{42}$.

Polyamine metabolism. Arginine serves as a precursor for polyamines, which are essential metabolites for cell proliferation ${ }^{50}$. Elevated levels of polyamines have been reported in cancer, and an extensive interplay between polyamines and mTOR signalling is beginning to emerge ${ }^{50}$. Arginine is cleaved by cytosolic arginase 1 (ARG1) or mitochondrial arginase 2 (ARG2) into ornithine and urea in the last step of the urea cycle. Ornithine decarboxylase (ODC), the rate-liming enzyme in polyamine biosynthesis and a MYC transcriptional target, then converts ornithine to putrescine, which is the immediate precursor for the polyamines spermidine and spermine ${ }^{51}$ (Fig. 2b). mTORC1 signalling might be able to promote this pathway because ODC translation correlates with eIF4E activity ${ }^{52}$, which is controlled by the mTORC1-4EBP axis (Fig. 2b). Also, mTORC1 was shown to stabilize ODC (also known as ODC1) mRNA in RAS-transformed cells by promoting the association of the stabilizing mRNA-binding protein HuR (also known as ELAVL1) to the ODC transcript ${ }^{53}$. Although polyamines promote proliferation and tumour growth, several enzymes of arginine and polyamine metabolism, including ASS1, ARG2 and ODC, are suppressed in clear cell RCC (ccRCC) $)^{54}$. Overexpression of ARG2 and ASS1 decreases arginine and aspartate levels, respectively, and thereby inhibits growth of ccRCC cells. ASS1 and/or ARG2 overexpression reduces tumour growth by various mechanisms, including reduction of mTORC1 activity ${ }^{54}$. The observed reduction in mTORC1 activity is most likely the direct consequence of reduced intracellular amino acid levels, including arginine, as observed in ccRCC xenografts upon ARG2 overexpression. The sequential synthesis of spermidine and spermine from putrescine requires aminopropyl groups, which are donated by decarboxylated S-adenosyl-methionine (decSAM) (Fig. 2b). The precursor of the decSAM-producing enzyme, 
adenosylmethionine decarboxylase 1 (AMD1), is stabilized by mTORC1 in a PTENdriven mouse model of prostate cancer ${ }^{55}$. In addition, AMD1 expression is reduced in patients with cancer who have been treated with the mTORC1 inhibitor everolimus ${ }^{55}$. As of yet, it has not been determined whether mTOR inhibition can synergize with polyamine pathway inhibition to reduce tumour growth. This will be particularly interesting to investigate, as so far, numerous drugs targeting polyamine metabolism have been developed but have not yet advanced beyond phase II clinical trials ${ }^{50}$.

\section{Branched chain amino acids}

The branched chain amino acids (BCAAs) leucine, iso- leucine and valine are essential amino acids in mammals. Cells control BCAA levels via uptake and protein or amino acid catabolism. BCAAs, especially leucine, activate mTORC1 by various mechanisms (Fig. 1).

The main BCAA transporter, SLC7A5, is highly expressed in many cancers including colorectal, liver and lung cancer ${ }^{56}$. It imports BCAAs and other essential amino acids in exchange for glutamine ${ }^{57}$. SLC7A5 expression, which is induced by oncogenic transcription factors, including NOTCH ${ }^{58}, \mathrm{MYC}^{59}$ and $\mathrm{HIF} 2 \alpha^{60}$, results in mTORC1 activation (Fig. 2c). For example, upregulation of SLC7A5 by HIF2 $\alpha$ activates mTORC1 in RCC-derived cell lines ${ }^{60}$. In a PTEN-negative mouse model of T cell acute lymphoblastic leukaemia, NOTCH- mediated SLC7A5 expression activates mTORC1, which in turn reprogrammes metabolism ${ }^{58}$. Interestingly, as shown in lymphoma cells, mTORC1 inhibition by rapamycin treatment reduces expression of various amino acid transporters including SLC7A5 $\left(\right.$ ref. $^{61}$ ).

On the basis of the above observations, leucine starvation has been investigated as a cancer treatment strategy. Leucine deprivation inhibits proliferation and induces apoptosis in cancer cell lines ${ }^{62}$. Biochemical or pharmacological inhibition of leucine uptake in metastatic castration-resistant prostate cancer cell lines inhibits mTORC1 signalling ${ }^{63}$. Some prostate cancers rely on androgen receptor (AR)-mediated SLC43A1 expression for leucine uptake ${ }^{63}$. In line with the role of leucine as an mTORC1 activator, anti-androgen treatment reduces both SLC43A1 expression and leucine levels, thereby decreasing mTORC1 activity. Lower intracellular leucine levels can elicit compensatory upregulation of SLC7A5 $\left(\right.$ ref. $\left.^{63}\right)$. Such a feedback mechanism could normalize leucine levels and reactivate mTORC1. This possibly explains why the combination of rapamycin and anti-androgen therapy is more effective than either agent alone, as observed in a PTEN-deficient prostate cancer mouse mode ${ }^{64}$.

Branched chain amino acid catabolism. Once taken up into the cell, BCAAs can be used for the synthesis of biomass or catabolized to provide nitrogen and carbon groups. First, branched chain aminotransferase 1 (BCAT1) and BCAT2 catalyse the conversion (a transamination) of BCAAs to branched chain ketoacids (BCKAs) (Fig. 2c). BCKAs are then subject to irreversible oxidative decarboxylation by the BCKA dehydro- genase (BCKD) complex. Further oxidation ultimately leads to the formation of acetyl-CoA and succinyl-CoA to fuel the TCA cycle or to contribute substrates for fatty acid synthesis (Fig. 2c). BCAT1 levels are upregulated in GBM, HCC, breast cancer, colorectal cancer and chronic myeloid leukaemia $(\mathrm{CML})^{65-69}$. However, the BCATs are 
bidirectional enzymes (can also convert BCKAs to BCAAs), and either direction may be important in cancer cells. In GBM, BCAT1 predominantly deaminates BCAAs to produce BCKAs and glutamate ${ }^{65}$. By contrast, in breast cancer and CML cells, BCAT1 uses BCKAs and glutamate to regenerate BCAAs and to activate mTORC1 ( refs $^{67,69}$ ). These findings indicate that increased BCAT1 expression alone is not sufficient to indicate the use of mTORC1 inhibitors, as sustained mTORC1 activation depends on the direction of BCAT1 function, and specific BCAT inhibitors have not been developed yet.

\section{mTOR signalling and glucose metabolism}

Glucose is the main source of cellular energy. Cancer cells increase glucose uptake and increase glycolytic flux to sustain growth and proliferation. In addition, aerobic glycolysis, a hallmark of cancer cells, provides a source of carbon moieties for anabolic processes including amino acid, lipid and nucleotide synthesis ${ }^{70}$.

\section{Glucose uptake and glycolysis}

mTOR signalling reprogrammes glucose metabolism by increasing the expression of glucose transporters and glycolytic enzymes ${ }^{71-73}$. Glucose transporter 1 (GLUT1; also known as SLC2A1) expression is increased in many cancers, including HCC and pancreatic, breast, lung and colorectal cancers ${ }^{74}$. In these cancers, mTOR signalling is also frequently activated ${ }^{3}$. mTOR signalling may activate GLUT1 expression via the transcription factors $\mathrm{HIF} 1 \alpha$ and $\mathrm{MYC}^{71-73}$.

Glycolysis converts glucose to pyruvate (Fig. 3). In the first step of glycolysis, hexokinase 2 (HK2) phosphorylates glucose to produce glucose-6-phosphate (G6P). Cancer cells often upregulate glycolysis by increasing HK2 expression or activity ${ }^{75}$. In prostate cancer cells, mTORC1 promotes glycolysis by increasing $\mathrm{HK} 2$ translation ${ }^{76}$. HK2 expression is also controlled at the transcriptional level by HIF1 $\alpha$ and $\mathrm{MYC}^{71}$. Furthermore, MYC induces expression of several other glycolytic enzymes including phosphoglucoisomerase (PGI), phosphofructokinase (PFK) and enolase (ENO) ${ }^{71}$ (Fig. 3). mTORC2, via AKT, phosphorylates HK2 to promote its association with mitochondria ${ }^{77}$. In GBM, mTORC2 increases HK2 activity $^{75}$. mTORC2 also promotes glycolysis via inhibition of FOXO1 or FOXO3 and consequential activation of MYC, as shown in $\mathrm{GBM}^{73}$ (Fig. 3). The dependence of cancer cells on glycolysis led to the development of inhibitors of HK2. The glycolytic inhibitor 2-deoxyglucose (2-DG), of which the phosphorylated form inhibits HK2, increased patient survival in a phase I clinical trial for advanced solid tumours ${ }^{78}$. Recently, synergistic effects of 2-DG with inhibitors of MYC, PI3K or mTOR have been reported in lymphoma cell lines ${ }^{7} 9$.

\section{Pyruvate kinase}

Pyruvate kinase catalyses the formation of pyruvate, the final product of glycolysis (Fig. 3 ). The tumour-specific isoform of pyruvate kinase, pyruvate kinase muscle isoform 2 (PKM2), is upregulated in many cancers, including lung cancer, neuroblastoma, RCC and gastric cancer ${ }^{80}$. Knockdown of PKM2 inhibits proliferation in both in vitro and in vivo cancer models ${ }^{80,81}$. Of note, PKM2 is regulated by mTORC1 but also, via a positive feedback loop, stimulates mTORC1, thereby amplifying mTORC1 signalling 
in cancer. MYC promotes PKM2 expression, to maintain glycolytic flux even under normoxia, in an mTORC1-dependent manner through heterogeneous nuclear ribonucleoprotein-dependent PKM2 mRNA splicing ${ }^{82}$. In turn, PKM2 activates mTORC1 through phosphorylation of the mTORC1 inhibitor prolinerich AKT substrate 1 (PRAS40). Phosphorylated PRAS40 dissociates from RAPTOR, resulting in activation of mTORC1 (ref. ${ }^{83}$ ). Furthermore, serine, which acts as an allosteric activator of PKM2, sustains mTORC1 activity, at least in lung cancer cells. When serine is depleted, PKM2 is inhibited, and glycolytic intermediates consequently accumulate and are diverted to de novo serine synthesis. Thus, PKM2 also contributes to mTORC1 activation by promoting de novo serine synthesis ${ }^{84}$. In addition, PKM2 not only is transcriptionally controlled by HIF1 $\alpha$ downstream of mTORC $1\left(\right.$ ref. $^{85}$ ) but also regulates HIF1 $\alpha$ transcriptional activity. PKM2 moonlights as a transcription coactivator that increases HIF $1 \alpha$ target gene binding. This creates a feedforward loop to sustain glycolysis in cancer cells ${ }^{86}$. Thus, mTORC1 and PKM2 might synergize to promote cell proliferation by driving glycolysis. Treatment with the PKM2 inhibitor shikonin shows promising results in advanced bladder cancer ${ }^{87}$ and gastric cancer cells both in vitro and in vivo ${ }^{88}$. However, a PKM2 inhibitor has not been tested in clinical trials, either alone or in combination with an mTORC1 inhibitor.

\section{Lactate and metabolic symbiosis}

Glycolytic cancer cells produce high amounts of pyruvate, which is converted to lactate by the bidirectional enzyme lactate dehydrogenase (LDH) (Fig. 3). LDH, via the regeneration of NAD+ during the conversion of pyruvate to lactate, allows cancer cells to sustain glycolysis ${ }^{71}$.

This differential use of lactate and glucose can be observed within a tumour in a phenomenon known as metabolic symbiosis ${ }^{91,92}$. For example, hypoxic cancer cells distant from blood vessels import glucose, via GLUT1, to perform glycolysis ${ }^{91,92}$ and secrete excess lactate via monocarboxylate transporter 4 (MCT4). In turn, normoxic cancer cells in close proximity to blood vessels take up lactate via MCT1 to fuel the TCA cycle for ATP generation and for synthesis of amino acids, which leads to mTORC1 activation ${ }^{91,92}$ (box 1). Interestingly, mTORC1 inhibition by rapamycin disrupts metabolic symbiosis in this setting, leading to increased glucose uptake and glycolysis in normoxic cells and glucose deprivation and cell death in hypoxic cells ${ }^{91}$. This suggests that metabolic symbiosis might be targeted for anticancer therapy. For example, the MCT1 inhibitor AZD-3965 is currently being tested in patients with prostate cancer, gastric cancer and diffuse large B cell lymphoma ${ }^{93}$ (Table 1). Because mTOR promotes glycolysis and this can increase lactate flux, combination therapies of mTORC1 inhibitors with LDH or MCT inhibitors should be considered.

\section{mTOR and nucleotide metabolism}

Cancer cells have an increased demand for nucleotides. Nucleotides can be synthesized de novo or generated by the salvage pathway that recycles degradation intermediates of pre-existing nucleotides ${ }^{38}$. Formation of the pyrimidine and purine rings in nucleotides requires amino acids and ribose-5-phosphoribosyl-1-pyrophosphate (PRPP), among other substrates ${ }^{38}$ (Fig. 4). PRPP is generated from ribose-5-phosphate (R5P), which is 
produced in the pentose phosphate pathway (PPP), a pathway that is also under control of mTOR signalling.

\section{Pentose phosphate pathway}

The PPP metabolizes G6P and other intermediates of the glycolytic pathway in its oxidative and non- oxidative branches ${ }^{94}$ (Fig. 4). The oxidative PPP branch uses G6P to produce ribulose-5-phosphate and NADPH, for which the rate- limiting enzyme is G6P dehydrogenase (G6PD) ${ }^{94}$. G6PD levels and activity are elevated in many cancers, including gastric cancer, RCC, breast cancer, lung cancer and others95. The nonoxidative branch produces R5P, which is converted to PRPP by the enzyme phosphoribosyl pyrophosphate synthase 1 (PRPS1) or PRPS2 $\left(\right.$ ref. $^{94}$ ). Both mTORC1 and mTORC2 signalling promote the PPP. mTORC2 elevates G6P levels to fuel the PPP via AKT- mediated phosphorylation and activation of HK2 (ref. ${ }^{77}$ ) (Fig. 3). mTORC1 stimulates flux through both branches of the PPP by increasing expression of at least two PPP enzymes (Fig. 4). In an HCC model, mTORC1 increases flux through the PPP both by stimulating glycolysis, which provides PPP substrates, and by increasing levels of G6PD96 and R5P isomerase A (RPIA) (which generates R5P from ribulose-5-phosphate $)^{96}$. As shown in mouse embryonic fibroblasts, mTORC1 stimulates the PPP most likely through S6K- mediated activation of the transcription factors HIF1 $\alpha$ and SREBP1 (ref. ${ }^{97}$ ) (Fig. 4). Consistent with this observation, PI3K signalling stimulates flux through both branches of the PPP in PTEN- deficient breast cancer models ${ }^{98}$.

\section{Purine metabolism}

Synthesis of the purine ring requires glutamine, glycine, aspartate, formyltetrahydrofolate (fTHF) and $\mathrm{CO}_{2}$. Purine rings are assembled directly on PRPP, resulting in the formation of inosine monophosphate (IMP) (Fig. 4). mTOR signalling stimulates purine synthesis indirectly, mainly by promoting enzymes that deliver substrates for the assembly of purine nucleotides. PRPS2 is the rate-limiting enzyme in purine synthesis and also provides PRPP for pyrimidine synthesis. mTORC1 and MAPK kinase 1 and/or MAPK kinase 2 induce PRPS2 expression through MYC and eIF4E99. mTORC1 also promotes purine nucleotide synthesis by upregulating the mitochondrial enzyme methylenetetrahydrofolate dehydrogenase 2 (MTHFD2) ${ }^{100}$. MTHFD2 mediates the formation of $\mathrm{fTHF}$, which donates carbon moieties for purine synthesis.

Interestingly, purine nucleotides also regulate mTORC1 activity. Short-term deprivation of adenylate, but not guanylate, inhibits mTORC1 (ref. ${ }^{101}$ ). This inhibition (or activation after restoring adenylate levels) is TSC-dependent but RAG-independent and AMPactivated kinase (AMPK)-independent ${ }^{101}$. Long-term guanylate starvation inhibits mTORC1 activity ${ }^{101,102}$, but the underlying mechanism is unclear.

The mutual determinism of purine synthesis and mTOR activity is reflected in studies exploring pharmacological inhibition of the purine biosynthetic enzyme glycinamide ribonucleotide formyltransferase (GARFT; also known as GART) by AG2037. GARFT 
inhibition results in tumour growth suppression in NSCLC xenografts, at least in part by inhibition of mTORC1 signalling102. Furthermore, mTORC1-dependent cancer cells have an elevated demand for nucleotides, and this vulnerability can be exploited by inhibition of guanine nucleotide synthesis. However, mizoribine, an IMP dehydrogenase (IMPDH) inhibitor, causes cancer cell death as a result of sustained mTORC 1 activity in the absence of nucleotide synthesis ${ }^{103}$. Thus, in this situation, inhibition of guanine nucleotide synthesis alone is sufficient, and addition of an mTORC1 inhibitor would even be counterproductive ${ }^{103}$.

\section{Pyrimidine metabolism}

The pyrimidine ring is assembled before it is condensed with PRPP. The initial steps of pyrimidine synthesis are carried out by the trifunctional enzyme carbamoyl phosphate synthetase 2, aspartate transcarbamylase and dihydroorotase (CAD) (Fig. 4), which uses glutamine, aspartate, bicarbonate ( $\mathrm{HCO}^{-}$) and ATP for the formation of dihydroorotate (DHO). DHO dehydrogenase (DHOD) converts DHO to orotate, which combines with PRPP to form orotate monophosphate (OMP), the precursor for all pyrimidine nucleotides (Fig. 4). CAD is a MYC target ${ }^{104}$ and is post-translationally regulated by mTORC1. The mTORC1 effector $\mathrm{S} 6 \mathrm{~K}$ phosphorylates $\mathrm{CAD}$ to promote CAD oligomerization and ultimately pyrimidine synthesis ${ }^{105,106}$.

Interestingly, pyrimidine synthesis is also coupled to alterations in arginine metabolism. Carbamoyl phosphate is synthesized by the carbamoyl phosphate synthetase 2 moiety of CAD and by CPS1 in mitochondria. CPS1 expression, like mTORC1 signalling, is inhibited by liver kinase B1 (LKB1; also known as STK11)-AMPK signalling 107,108 (Fig. 1). In NSCLC cells with oncogenic KRAS and loss of LKB1, mTORC1 is activated $^{107}$, and elevated CPS1 expression enables CPS1 to unconventionally produce carbamoyl phosphate for pyrimidine nucleotide synthesis ${ }^{108}$. Furthermore, the amino acid aspartate has emerged as another positive regulator of mTORC1 activity through its role in pyrimidine synthesis. Aspartate is used by ASS1 in the urea cycle and by CAD in pyrimidine synthesis. ASS1-deficient cancer cells are unable to use aspartate in the urea cycle, making more aspartate available for the production of pyrimidines ${ }^{41}$. Indeed, knockdown of ASS 1 in osteosarcoma cell lines increases intracellular aspartate levels, which promotes CAD activity and thereby increases pyrimidine synthesis and proliferation ${ }^{41}$. Therefore, combining inhibition of mTORC1 and inhibition of pyrimidine synthesis could be an effective cancer therapy. While this has not been tested directly, studies indicate that treatments that indirectly reduce mTORC1 activity can act synergistically with inhibition of pyrimidine synthesis. Indeed, a combination of ADI-PEG20 treatment (which decreases the levels of arginine ${ }^{109}$ and thereby mTORC1 activity, as arginine is a potent activator of $\mathrm{mTORC} 1$ ) and inhibition of pyrimidine synthesis by 5-flurouracil is synergistic in ASS1-deficient HCC cell lines ${ }^{110}$.

\section{mTOR and fatty acid and lipid metabolism}

\section{Fatty acid metabolism}

Cells acquire fatty acids through uptake or via de novo synthesis, and both processes are often upregulated in cancer ${ }^{111}$ (Fig. 5). Both fatty acid synthesis and fatty acid uptake 
are stimulated in response to mTOR signalling. As such, both mTORC1 and mTORC2 stimulate expression and proteolytic processing of SREBP1, the main transcription factor that induces fatty acid synthesis enzymes including ATP citrate lyase (ACLY), acetyl-CoA carboxylase 1 (ACC1), fatty acid synthase (FASN) and stearoyl-CoA desaturase 1 (SCD1; also known as SCD) and the fatty acid transporter CD36 (refs ${ }^{112,113,114,115}$ ) (Fig. 5). Furthermore, mTORC1 promotes SREBP1 activity by preventing the nuclear entry of LIPIN1, a negative regulator of SREBP1, in several cell lines, including a liver cancer cell line ${ }^{116}$. In renal angiomyolipoma cell lines, mTORC1-S6K signalling increases splicing and stability of FASN, ACLY and SCD1 transcripts through phosphorylation and activation of serine/arginine-rich protein kinase 2 (SRPK2) ${ }^{117}$. mTORC2, via its downstream effector AKT, promotes SREBP1 expression $^{114,118}$ and prevents SREBP1 degradation in cancer cells ${ }^{119}$. In mice deficient for both PTEN and TSC1, mTORC2 drives liver cancer, at least in part by activating SREBP1 (ref. ${ }^{118}$ ) (Fig. 5). Lastly, mTORC2 also phosphorylates and activates ACLY, thereby supporting the growth of HER2 (also known as ERBB2)-positive and PI3Kmutant breast cancer cell lines ${ }^{120}$.

Notably, MYC also regulates ACC1, FASN and SCD1 expression in cancer ${ }^{121}$. In addition, MYC promotes TCA cycle enzyme expression to increase the production of citrate, the precursor for de novo fatty acid synthesis ${ }^{122}$ (Fig. 5). Accordingly, the targeting of fatty acid synthesis has been exploited as an anticancer strategy, and inhibitors of FASN, the rate-limiting enzyme in fatty acid synthesis, have entered clinical trials. For example, TVB-2640, the first oral and selective FASN inhibitor, has recently entered clinical trials for several solid tumours, including colon cancer, breast cancer and astrocytoma ${ }^{123,124,125,126}$ (Table 1). Another FASN inhibitor, epigallocatechin gallate (EGCG), will be tested in patients with colorectal cancer ${ }^{127}$ (Table 1).

One obstacle in targeting FASN is that cancer cells might be able to circumvent inhibition of fatty acid synthesis by increasing fatty acid uptake through expression of fatty acid transporters such as CD36. Targeting mTOR signalling, because it promotes both fatty acid uptake and fatty acid synthesis, might be a strategy to overcome potential resistance mechanisms. mTOR inhibition alone might not be effective owing to mTORindependent inputs from other signalling pathways. These include MYC-mediated expression of fatty acid synthesis genes ${ }^{121}$, AKT-independent phosphorylation of ACLY $^{128}$ and induction of the fatty acid synthesis-promoting transcription factor carbohydrate responsive element binding protein (ChREBP), which is upregulated in certain cancers ${ }^{129}$. Thus, combining fatty acid synthesis and mTOR inhibitors might prove beneficial for patients with cancer. This notion is supported by a preclinical study that reported synergistic effects of the mTORC1 inhibitor rapamycin in combination with an FASN inhibitor in breast cancer cells ${ }^{130}$.

\section{Lipid metabolism}

Cancer cells require lipids, including glycerophospholipids, sphingolipids and sterols, for membrane synthesis and signalling (second messengers) (Fig. 5). The mTORcontrolled transcription factor SREBP1 stimulates expression of elongation of very long 
chain fatty acids (ELOVL) to promote elongation of polyunsaturated fatty acids, which are the immediate precursors for synthesis of many lipids ${ }^{131}$. mTORC1 promotes synthesis of the lipid phosphatidylcholine by activating the rate-limiting enzyme phosphocholine cytidylyltransferase- $\alpha$ (CCT $\alpha$; also known as PCYT1A $)^{132}$, which is also under the transcriptional control of SREBP1 (ref. ${ }^{131}$ ).

Sphingolipids, such as ceramides or sphingosine-1-phosphate (S1P), have important signalling functions (Fig. 5). Ceramides suppress cancer cell growth, in part by reducing AKT activity ${ }^{133}$. Cancer cells thus employ various mechanisms to prevent ceramide accumulation. These mechanisms include upregulation of glucosylceramide synthase (GCS) (which converts ceramide to glycosylceramide), among others ${ }^{134}$ (Fig. 5). In a PTEN-deficient and TSC1-deficient mouse model for HCC, mTORC2 stimulates tumorigenesis by promoting expression of GCS, thereby preventing ceramide accumulation. Knockdown of GCS in the mouse model prevents HCC tumorigenesis ${ }^{118}$.

In contrast to ceramide, S1P promotes cell proliferation and migration. Many cancers, including breast, colon, lung and renal carcinoma, increase sphingosine kinase 1 (SPHK1) expression, which leads to increased S1P levels ${ }^{135}$. SPHK1-S1P signalling, which is activated upon hypoxia, promotes cancer cell proliferation and migration, at least in part by activating mTOR signalling ${ }^{135,136}$.

Specific sphingolipid synthesis inhibitors are now being evaluated in preclinical and early-phase clinical trials as anticancer agents. Myriocin, a serine palmitoyltransferase (SPT) inhibitor, reduces proliferation of lung cancer and melanoma cell lines ${ }^{137}$ and attenuates HCC in mice ${ }^{118}$. Furthermore, the SPHK2 inhibitor ABC294640 is in clinical trials for cholangiocarcinoma ${ }^{138}$, multiple myeloma ${ }^{139}$ and advanced $\mathrm{HCC}^{140}$ (Table 1). Interestingly, ABC294640 reduces mTORC1 and mTORC2 activity in colorectal cancer cell lines and primary cancer cells, resulting in growth inhibition and apoptosis $^{141}$.

\section{Concluding remarks}

Notably, in addition to the process described above, recent work has revealed links between mTOR signalling and metabolism of amino acids that have not been recognized as activators of $\mathrm{mTORC1}$, that is, amino acids other than glutamine, arginine and leucine. For example, mTORC1 promotes serine biosynthesis ${ }^{100,142,143}$; mTORC2 regulates the cystine-glutamate antiporter xCT (also known as SLC7A11), which is upregulated in triple-negative breast cancer and $\mathrm{GBM}^{144}$, and a subset of cancer cells depends on proline uptake to sustain mTORC1-4EBP signalling ${ }^{145}$. In addition to amino acids, other metabolites have recently been described to modulate mTORC1 activity, including S-adenosyl-methionine (SAM) ${ }^{146}$, purine nucleotides ${ }^{101,102}$ and cholesterol ${ }^{147}$. Conversely, mTOR signalling regulates multiple aspects of nucleotide, fatty acid and lipid metabolism (see above). These findings further suggest that mTOR inhibitors could be considered for cancers with mTOR-related metabolic alterations. To pursue such a strategy, it is important to understand further the mutual determinism of metabolic reprogramming and mTOR signalling. It might also be necessary to revise 
our view of how driver mutations determine tumour development and metabolic alterations. A recently proposed concept is that the tissue of origin rather than underlying mutations dictates how cancer cells reprogramme their metabolism ${ }^{148}$. Therefore, it will be necessary to define the metabolic signatures for cancer subtypes to classify patients for treatment options. Such classifications might also reveal how metabolic vulnerabilities can be therapeutically exploited. For example, in LSCC, a metabolic signature has been suggested to predict responsiveness or resistance to mTOR inhibitors ${ }^{30}$. Several drugs, such as metformin, dichloracetate ${ }^{149}$ or disulfiram ${ }^{150}$, that interfere with cellular metabolism and have been used to treat metabolic diseases for many years are now being repurposed for cancer treatment. For example, metformin, the most frequently prescribed drug for the treatment of type 2 diabetes, activates AMPK, inhibits mTORC1 (ref. ${ }^{151}$ ) and attenuates protein synthesis, the TCA cycle and nucleotide synthesis ${ }^{152}$. Thus, metformin, alone or in combination with other cancer drugs, has been shown to be effective in reducing tumour growth in several models ${ }^{153-}$ ${ }^{155}$. Indeed, metformin is currently in a number of clinical trials, including in combination with mTORC1 inhibitors ${ }^{156-158}$ (Table 1).

Several clinical trials are now evaluating the use of mTOR inhibitors in combination with other targeted drugs that interfere with cancer cell metabolism. However, to optimize treatment strategies, we will need to understand how anticancer drugs targeting metabolism affect cancer and non- cancer cells in the tumour microenvironment, which could help, for example, to avoid drug- induced immunosuppression (Box 1). As such, therapeutic strategies that aim at arginine or glucose deprivation will also inhibit $\mathrm{T}$ cell activation and proliferation, thereby probably precluding an adequate immune response towards the tumour. Members of the first generation of mTOR inhibitors and drugs targeting metabolic alterations have had weak therapeutic efficacy as single agents (for extended reading on mTOR inhibitors, see refs ${ }^{18,159}$ for reviews). Secondgeneration mTOR inhibitors are currently being tested, and more drugs interfering with metabolic alterations are being developed. The extensive interplay between mTOR signalling and metabolic reprogramming strongly supports further exploration of combinations of drugs targeting metabolism with mTOR inhibitors.

\section{References}

1. Vander Heiden, M. G. \& DeBerardinis, R. J. Understanding the intersections between metabolism and cancer biology. Cell 168, 657-669 (2017).

2. Saxton, R. A. \& Sabatini, D. M. mTOR signaling in growth, metabolism, and disease. Cell 168, 960-976 (2017).

3. Zhang, Y. et al. A pan-cancer proteogenomic atlas of PI3K/AKT/mTOR pathway alterations. Cancer Cell 31, 820-832 (2017).

4. Alessi, D. R. et al. Characterization of a 3-phosphoinositide-dependent protein kinase which phosphorylates and activates protein kinase $\mathrm{B} \alpha$. Curr. Biol. 7, 261269 (1997).

5. Inoki, K., Li, Y., Zhu, T., Wu, J. \& Guan, K. L. TSC2 is phosphorylated and inhibited by Akt and suppresses mTOR signalling. Nat. Cell Biol. 4, 648-657 (2002). 
6. Inoki, K., Li, Y., Xu, T. \& Guan, K. L. Rheb GTPase is a direct target of TSC2 GAP activity and regulates mTOR signaling. Genes Dev. 17, 1829-1834 (2003).

7. Long, X., Lin, Y., Ortiz-Vega, S., Yonezawa, K. \& Avruch, J. Rheb binds and regulates the mTOR kinase. Curr. Biol. 15, 702-713 (2005).

8. Yang, $\mathrm{H}$. et al. Mechanisms of mTORC1 activation by RHEB and inhibition by PRAS40. Nature 552, 368-373 (2017).

9. Gonzalez, A. \& Hall, M. N. Nutrient sensing and TOR signaling in yeast and mammals. EMBO J. 36, 397-408 (2017).

10. Wolfson, R. L. \& Sabatini, D. M. The dawn of the age of amino acid sensors for the mTORC1 pathway. Cell Metab. 26, 301-309 (2017).

11. Wyant, G. A. et al. mTORC1 activator SLC38A9 is required to efflux essential amino acids from lysosomes and use protein as a nutrient. Cell 171, 642-654 (2017).

12. Duran, R. V. et al. Glutaminolysis activates Rag- mTORC1 signaling. Mol. Cell 47, 349-358 (2012).

13. Jewell, J. L. et al. Differential regulation of mTORC1 by leucine and glutamine. Science 347, 194-198 (2015).

14. Liu, P. et al. PtdIns(3,4,5)P3-dependent activation of the mTORC2 kinase complex. Cancer Discov. 5, 1194-1209 (2015).

15. Zinzalla, V., Stracka, D., Oppliger, W. \& Hall, M. N. Activation of mTORC2 by association with the ribosome. Cell 144, 757-768 (2011).

16. Thorpe, L. M., Yuzugullu, H. \& Zhao, J. J. PI3K in cancer: divergent roles of isoforms, modes of activation and therapeutic targeting. Nat. Rev. Cancer 15, $7-$ 24 (2015).

17. Mayer, I. A. \& Arteaga, C. L. The PI3K/AKT pathway as a target for cancer treatment. Annu. Rev. Med. 67, 11-28 (2016).

18. Benjamin, D., Colombi, M., Moroni, C. \& Hall, M. N. Rapamycin passes the torch: a new generation of mTOR inhibitors. Nat. Rev. Drug Discov. 10, 868880 (2011).

19. Wise, D. R. \& Thompson, C. B. Glutamine addiction: a new therapeutic target in cancer. Trends Biochem. Sci. 35, 427-433 (2010).

20. Yang, L., Venneti, S. \& Nagrath, D. Glutaminolysis: a hallmark of cancer metabolism. Annu. Rev. Biomed. Eng. 19, 163-194 (2017).

21. Bhutia, Y. D., Babu, E., Ramachandran, S. \& Ganapathy, V. Amino acid transporters in cancer and their relevance to "glutamine addiction": novel targets for the design of a new class of anticancer drugs. Cancer Res. 75, 1782-1788 (2015).

22. Lu, J. et al. Effects of targeting SLC1A5 on inhibiting gastric cancer growth and tumor development in vitro and in vivo. Oncotarget 8, 76458-76467 (2017).

23. Wang, Q. et al. Targeting ASCT2-mediated glutamine uptake blocks prostate cancer growth and tumour development. J. Pathol. 236, 278-289 (2015).

24. van Geldermalsen, M. et al. ASCT2/SLC1A5 controls glutamine uptake and tumour growth in triple- negative basal-like breast cancer. Oncogene 35, 32013208 (2016).

25. Broer, A., Rahimi, F. \& Broer, S. Deletion of amino acid transporter ASCT2 
(SLC1A5) reveals an essential role for transporters SNAT1 (SLC38A1) and SNAT2 (SLC38A2) to sustain glutaminolysis in cancer cells. J. Biol. Chem. 291, 13194-13205 (2016).

26. Csibi, A. et al. The mTORC1/S6K1 pathway regulates glutamine metabolism through the eIF4B-dependent control of c-Myc translation. Curr. Biol. 24, 22742280 (2014).

27. Gao, P. et al. c-Myc suppression of miR-23a/b enhances mitochondrial glutaminase expression and glutamine metabolism. Nature 458, 762-765 (2009).

28. Csibi, A. et al. The mTORC1 pathway stimulates glutamine metabolism and cell proliferation by repressing SIRT4. Cell 153, 840-854 (2013).

29. van der Vos, K. E. et al. Modulation of glutamine metabolism by the PI(3)KPKB-FOXO network regulates autophagy. Nat. Cell Biol. 14, 829-837 (2012).

30. Momcilovic, M. et al. The GSK3 signaling axis regulates adaptive glutamine metabolism in lung squamous cell carcinoma. Cancer Cell 33, 905-921 (2018).

31. Schulte, M. L. et al. Pharmacological blockade of ASCT2-dependent glutamine transport leads to antitumor efficacy in preclinical models. Nat. Med. 24, 194202 (2018).

32. US National Library of Medicine. ClinicalTrials.gov https://clinicaltrials.gov/ct2/show/NCT03057600 (2018).

33. US National Library of Medicine. ClinicalTrials.gov https://clinicaltrials.gov/ct2/show/NCT02071862 (2018).

34. Lu, Y. et al. Overexpression of arginine transporter CAT-1 is associated with accumulation of L-arginine and cell growth in human colorectal cancer tissue. PLOS ONE 8, e73866 (2013).

35. Abdelmagid, S. A., Rickard, J. A., McDonald, W. J., Thomas, L. N. \& Too, C. K. CAT-1-mediated arginine uptake and regulation of nitric oxide synthases for the survival of human breast cancer cell lines. J. Cell. Biochem. 112, 1084-1092 (2011).

36. Kishikawa, T. et al. Decreased miR122 in hepatocellular carcinoma leads to chemoresistance with increased arginine. Oncotarget 6, 8339-8352 (2015).

37. Masuda, M. et al. Alternative mammalian target of rapamycin (mTOR) signal activation in sorafenib- resistant hepatocellular carcinoma cells revealed by array-based pathway profiling. Mol. Cell. Proteomics 13, 1429-1438 (2014).

38. Luengo, A., Gui, D. Y. \& Vander Heiden, M. G. Targeting metabolism for cancer therapy. Cell Chem. Biol. 24, 1161-1180 (2017).

39. Dillon, B. J. et al. Incidence and distribution of argininosuccinate synthetase deficiency in human cancers: a method for identifying cancers sensitive to arginine deprivation. Cancer 100, 826-833 (2004).

40. Delage, B. et al. Arginine deprivation and argininosuccinate synthetase expression in the treatment of cancer. Int. J. Cancer 126, 2762-2772 (2010).

41. Rabinovich, S. et al. Diversion of aspartate in ASS1-deficient tumours fosters de novo pyrimidine synthesis. Nature 527, 379-383 (2015). This paper shows that loss of ASS1 expression promotes pyrimidine biosynthesis by aspartate accumulation.

42. Tsai, W. B. et al. Activation of Ras/PI3K/ERK pathway induces c-Myc 
stabilization to upregulate argininosuccinate synthetase, leading to arginine deiminase resistance in melanoma cells. Cancer Res. 72, 2622-2633 (2012).

43. Patil, M. D., Bhaumik, J., Babykutty, S., Banerjee, U. C. \& Fukumura, D. Arginine dependence of tumor cells: targeting a chink in cancer's armor. Oncogene 35, 4957-4972 (2016).

44. US National Library of Medicine. ClinicalTrials.gov https://clinicaltrials.gov/ct2/show/NCT02899286 (2017).

45. US National Library of Medicine. ClinicalTrials.gov https://clinicaltrials.gov/ct2/show/NCT02285101 (2018).

46. US National Library of Medicine. ClinicalTrials.gov https://clinicaltrials.gov/ct2/show/NCT01497925 (2018).

47. US National Library of Medicine. ClinicalTrials.gov https://clinicaltrials.gov/ct2/show/NCT03254732 (2018).

48. Shen, W. et al. A novel and promising therapeutic approach for NSCLC: recombinant human arginase alone or combined with autophagy inhibitor. Cell Death Dis. 8, e2720 (2017).

49. Tsai, W. B. et al. Resistance to arginine deiminase treatment in melanoma cells is associated with induced argininosuccinate synthetase expression involving cMyc/HIF-1alpha/Sp4. Mol. Cancer Ther. 8, 3223-3233 (2009).

50. Casero, R. A. Jr, Murray Stewart, T. \& Pegg, A. E. Polyamine metabolism and cancer: treatments, challenges and opportunities. Nat. Rev. Cancer 18, 681-695 (2018).

51. Bello-Fernandez, C., Packham, G. \& Cleveland, J. L. The ornithine decarboxylase gene is a transcriptional target of c-Myc. Proc. Natl Acad. Sci. USA 90, 7804-7808 (1993).

52. Rousseau, D., Kaspar, R., Rosenwald, I., Gehrke, L. \& Sonenberg, N. Translation initiation of ornithine decarboxylase and nucleocytoplasmic transport of cyclin D1 mRNA are increased in cells overexpressing eukaryotic initiation factor 4E. Proc. Natl Acad. Sci. USA 93, 1065-1070 (1996).

53. Origanti, S. et al. Ornithine decarboxylase mRNA is stabilized in an mTORC1dependent manner in Ras-transformed cells. Biochem. J. 442, 199-207 (2012).

54. Ochocki, J. D. et al. Arginase 2 suppresses renal carcinoma progression via biosynthetic cofactor pyridoxal phosphate depletion and increased polyamine toxicity. Cell Metab. 27, 1263-1280.e6 (2018).

55. Zabala-Letona, A. et al. mTORC1-dependent AMD1 regulation sustains polyamine metabolism in prostate cancer. Nature 547, 109-113 (2017). This paper reveals that $\mathrm{mTORC} 1$ promotes polyamine biosynthesis by stabilization of (pro)AMD1.

56. Wang, Q. \& Holst, J. L-type amino acid transport and cancer: targeting the mTORC1 pathway to inhibit neoplasia. Am. J. Cancer Res. 5, 1281-1294 (2015).

57. Nicklin, P. et al. Bidirectional transport of amino acids regulates mTOR and autophagy. Cell 136, 521-534 (2009).

58. Grzes, K. M. et al. Control of amino acid transport coordinates metabolic reprogramming in T cell malignancy. Leukemia 31, 2771-2779 (2017). 
59. Yue, M., Jiang, J., Gao, P., Liu, H. \& Qing, G. Oncogenic MYC activates a feedforward regulatory loop promoting essential amino acid metabolism and tumorigenesis. Cell Rep. 21, 3819-3832 (2017).

60. Elorza, A. et al. HIF2alpha acts as an mTORC1 activator through the amino acid carrier SLC7A5. Mol. Cell 48, 681-691 (2012)

61. Peng, T., Golub, T. R. \& Sabatini, D. M. The immunosuppressant rapamycin mimics a starvation-like signal distinct from amino acid and glucose deprivation. Mol. Cell. Biol. 22, 5575-5584 (2002).

62. Xiao, F. et al. Leucine deprivation inhibits proliferation and induces apoptosis of human breast cancer cells via fatty acid synthase. Oncotarget 7, 63679-63689 (2015).

63. Wang, Q. et al. Androgen receptor and nutrient signaling pathways coordinate the demand for increased amino acid transport during prostate cancer progression. Cancer Res. 71, 7525-7536 (2011).

64. Zhang, W. et al. Inhibition of tumor growth progression by antiandrogens and mTOR inhibitor in a Pten-deficient mouse model of prostate cancer. Cancer Res. 69, 7466-7472 (2009).

65. Tonjes, M. et al. BCAT1 promotes cell proliferation through amino acid catabolism in gliomas carrying wild-type IDH1. Nat. Med. 19, 901-908 (2013).

66. $\mathrm{Xu}, \mathrm{M}$. et al. BCAT1 promotes tumor cell migration and invasion in hepatocellular carcinoma. Oncol. Lett. 12, 2648-2656 (2016).

67. Zhang, L. \& Han, J. Branched-chain amino acid transaminase 1 (BCAT1) promotes the growth of breast cancer cells through improving mTOR-mediated mitochondrial biogenesis and function. Biochem. Biophys. Res. Commun. 486, 224-231 (2017).

68. Yoshikawa, R. et al. ECA39 is a novel distant metastasis-related biomarker in colorectal cancer. World J. Gastroenterol. 12, 5884-5889 (2006).

69. Hattori, A. et al. Cancer progression by reprogrammed BCAA metabolism in myeloid leukaemia. Nature 545, 500-504 (2017).

70. Vander Heiden, M. G., Cantley, L. C. \& Thompson, C. B. Understanding the Warburg effect: the metabolic requirements of cell proliferation. Science 324, 1029-1033 (2009).

71. Tran, Q., Lee, H., Park, J., Kim, S. H. \& Park, J. Targeting cancer metabolism revisiting the Warburg effects. Toxicol. Res. 32, 177-193 (2016).

72. Buller, C. L. et al. A GSK-3/TSC2/mTOR pathway regulates glucose uptake and GLUT1 glucose transporter expression. Am. J. Physiol. Cell Physiol. 295, C836C843 (2008).

73. Masui, K. et al. mTOR complex 2 controls glycolytic metabolism in glioblastoma through FoxO acetylation and upregulation of c-Myc. Cell Metab. 18, 726-739 (2013). This paper demonstrates how mTORC2 controls glucose metabolism via FOXO and MYC in GBM.

74. Carvalho, K. C. et al. GLUT1 expression in malignant tumors and its use as an immunodiagnostic marker. Clinics 66, 965-972 (2011).

75. Wolf, A. et al. Hexokinase 2 is a key mediator of aerobic glycolysis and promotes tumor growth in human glioblastoma multiforme. J. Exp. Med. 208, 
313-326 (2011).

76. Wang, L. et al. Hexokinase 2-mediated Warburg effect is required for PTENand p53-deficiency-driven prostate cancer growth. Cell Rep. 8, 1461-1474 (2014).

77. Betz, C. et al. mTOR complex 2-Akt signaling at mitochondria-associated endoplasmic reticulum membranes (MAM) regulates mitochondrial physiology. Proc. Natl Acad. Sci. USA 110, 12526-12534 (2013).

78. Raez, L. E. et al. A phase I dose-escalation trial of 2-deoxy-D-glucose alone or combined with docetaxel in patients with advanced solid tumors. Cancer Chemother. Pharmacol. 71, 523-530 (2013).

79. Broecker-Preuss, M., Becher-Boveleth, N., Bockisch, A., Duhrsen, U. \& Muller, S. Regulation of glucose uptake in lymphoma cell lines by c-MYC- and PI3Kdependent signaling pathways and impact of glycolytic pathways on cell viability. J. Transl Med. 15, 158 (2017).

80. Wang, C. et al. PKM2 promotes cell migration and inhibits autophagy by mediating PI3K/AKT activation and contributes to the malignant development of gastric cancer. Sci. Rep. 7, 2886 (2017).

81. Goldberg, M. S. \& Sharp, P. A. Pyruvate kinase M2-specific siRNA induces apoptosis and tumor regression. J. Exp. Med. 209, 217-224 (2012).

82. Sun, Q. et al. Mammalian target of rapamycin up-regulation of pyruvate kinase isoenzyme type M2 is critical for aerobic glycolysis and tumor growth. Proc. Natl Acad. Sci. USA 108, 4129-4134 (2011).

83. He, C. L. et al. Pyruvate kinase M2 activates mTORC1 by phosphorylating AKT1S1. Sci. Rep. 6, 21524 (2016).

84. Ye, J. et al. Pyruvate kinase M2 promotes de novo serine synthesis to sustain mTORC1 activity and cell proliferation. Proc. Natl Acad. Sci. USA 109, 69046909 (2012).

85. Lynch, T., Moloughney, J. G. \& Jacinto, E. in PI3K-mTOR in Cancer and Cancer Therapy - Cancer Drug Discovery and Development (eds Dey, N., De, P. \& Leyland-Jones, B.) 29-63 (Humana Press, 2016).

86. Luo, W. et al. Pyruvate kinase M2 is a PHD3- stimulated coactivator for hypoxia-inducible factor 1. Cell 145, 732-744 (2011).

87. Wang, X., Zhang, F. \& Wu, X. R. Inhibition of pyruvate kinase M2 markedly reduces chemoresistance of advanced bladder cancer to cisplatin. Sci. Rep. 7, 45983 (2017).

88. Liang, W. et al. Shikonin induces mitochondria- mediated apoptosis and enhances chemotherapeutic sensitivity of gastric cancer through reactive oxygen species. Sci. Rep. 6, 38267 (2016).

89. Faubert, B. et al. Lactate metabolism in human lung tumors. Cell 171, 358-371 (2017).

90. Hui, S. et al. Glucose feeds the TCA cycle via circulating lactate. Nature 551, 115-118 (2017).

91. Allen, E. et al. Metabolic symbiosis enables adaptive resistance to antiangiogenic therapy that is dependent on mTOR signaling. Cell Rep. 15, 11441160 (2016). 
92. Pisarsky, L. et al. Targeting metabolic symbiosis to overcome resistance to antiangiogenic therapy. Cell Rep. 15, 1161-1174 (2016).

93. US National Library of Medicine. ClinicalTrials.gov https://clinicaltrials.gov/ct2/show/NCT01791595 (2018).

94. Patra, K. C. \& Hay, N. The pentose phosphate pathway and cancer. Trends Biochem. Sci. 39, 347-354 (2014).

95. Wang, J. et al. Overexpression of G6PD is associated with poor clinical outcome in gastric cancer. Tumour Biol. 33, 95-101 (2012).

96. Evert, M. et al. V-AKT murine thymoma viral oncogene homolog/mammalian target of rapamycin activation induces a module of metabolic changes contributing to growth in insulin-induced hepatocarcinogenesis. Hepatology 55, 1473-1484 (2012).

97. Duvel, K. et al. Activation of a metabolic gene regulatory network downstream of mTOR complex 1. Mol. Cell 39, 171-183 (2010).

98. Juvekar, A. et al. Phosphoinositide 3-kinase inhibitors induce DNA damage through nucleoside depletion. Proc. Natl Acad. Sci. USA 113, 4338-4347 (2016).

99. Cunningham, J. T., Moreno, M. V., Lodi, A., Ronen, S. M. \& Ruggero, D. Protein and nucleotide biosynthesis are coupled by a single rate-limiting enzyme, PRPS2, to drive cancer. Cell 157, 1088-1103 (2014).

100. Ben-Sahra, I., Hoxhaj, G., Ricoult, S. J. H., Asara, J. M. \& Manning, B. D. mTORC1 induces purine synthesis through control of the mitochondrial tetrahydrofolate cycle. Science 351, 728-733 (2016).

101. Hoxhaj, G. et al. The mTORC1 signaling network senses changes in cellular purine nucleotide levels. Cell Rep. 21, 1331-1346 (2017).

102. Emmanuel, N. et al. Purine nucleotide availability regulates mTORC1 activity through the Rheb GTPase. Cell Rep. 19, 2665-2680 (2017).

103. Valvezan, A. J. et al. mTORC1 couples nucleotide synthesis to nucleotide demand resulting in a targetable metabolic vulnerability. Cancer Cell 32, 624638 (2017).

104. Miltenberger, R. J., Sukow, K. A. \& Farnham, P. J. An E-box-mediated increase in cad transcription at the G1/S-phase boundary is suppressed by inhibitory cMyc mutants. Mol. Cell. Biol. 15, 2527-2535 (1995).

105. Ben-Sahra, I., Howell, J. J., Asara, J. M. \& Manning, B. D. Stimulation of de novo pyrimidine synthesis by growth signaling through mTOR and S6K1. Science 339, 1323-1328 (2013).

106. Robitaille, A. M. et al. Quantitative phosphoproteomics reveal mTORC1 activates de novo pyrimidine synthesis. Science 339, 1320-1323 (2013).

107. Shackelford, D. B. \& Shaw, R. J. The LKB1-AMPK pathway: metabolism and growth control in tumour suppression. Nat. Rev. Cancer 9, 563-575 (2009).

108. Kim, J. et al. CPS1 maintains pyrimidine pools and DNA synthesis in KRAS/LKB1-mutant lung cancer cells. Nature 546, 168-172 (2017). This paper reports that CPS1 maintains pyrimidine synthesis in LKB1-mutant lung cancer.

109. Izzo, F. et al. Pegylated arginine deiminase treatment of patients with unresectable hepatocellular carcinoma: results from phase I/II studies. J. Clin. 
Oncol. 22, 1815-1822 (2004).

110. Thongkum, A. et al. The combination of arginine deprivation and 5-fluorouracil improves therapeutic efficacy in argininosuccinate synthetase negative hepatocellular carcinoma. Int. J. Mol. Sci. 18, 1175 (2017).

111. Baenke, F., Peck, B., Miess, H. \& Schulze, A. Hooked on fat: the role of lipid synthesis in cancer metabolism and tumour development. Dis. Model. Mech. 6, 1353-1363 (2013).

112. Owen, J. L. et al. Insulin stimulation of SREBP-1c processing in transgenic rat hepatocytes requires p70 S6-kinase. Proc. Natl Acad. Sci. USA 109, 1618416189 (2012).

113. Yecies, J. L. et al. Akt stimulates hepatic SREBP1c and lipogenesis through parallel mTORC1-dependent and independent pathways. Cell Metab. 14, 21-32 (2011).

114. Hagiwara, A. et al. Hepatic mTORC2 activates glycolysis and lipogenesis through Akt, glucokinase, and SREBP1c. Cell Metab. 15, 725-738 (2012).

115. Ricoult, S. J., Yecies, J. L., Ben-Sahra, I. \& Manning, B. D. Oncogenic PI3K and K-Ras stimulate de novo lipid synthesis through mTORC1 and SREBP. Oncogene 35, 1250-1260 (2016).

116. Peterson, T. R. et al. mTOR complex 1 regulates lipin 1 localization to control the SREBP pathway. Cell 146, 408-420 (2011).

117. Lee, G. et al. Post-transcriptional regulation of de novo lipogenesis by mTORC1S6K1-SRPK2 signaling. Cell 171, 1545-1558 (2017).

118. Guri, Y. et al. mTORC2 promotes tumorigenesis via lipid synthesis. Cancer Cell 32, 807-823 (2017). This paper reveals an essential role of mTORC2 in HCC through lipid synthesis.

119. Li, S., Oh, Y. T., Yue, P., Khuri, F. R. \& Sun, S. Y. Inhibition of mTOR complex 2 induces GSK3/FBXW7- dependent degradation of sterol regulatory elementbinding protein 1 (SREBP1) and suppresses lipogenesis in cancer cells. Oncogene 35, 642-650 (2016).

120. Chen, Y. et al. mTOR complex-2 stimulates acetyl-CoA and de novo lipogenesis through ATP citrate lyase in HER2/PIK3CA-hyperactive breast cancer.

Oncotarget 7, 25224-25240 (2016).

121. Stine, Z. E., Walton, Z. E., Altman, B. J., Hsieh, A. L. \& Dang, C. V. MYC, metabolism, and cancer. Cancer Discov. 5, 1024-1039 (2015).

122. Dang, C. V. MYC on the path to cancer. Cell 149, 22-35 (2012).

123. US National Library of Medicine. ClinicalTrials.gov https://clinicaltrials.gov/ct2/show/NCT02980029 (2018).

124. US National Library of Medicine. ClinicalTrials.gov https://clinicaltrials.gov/ct2/show/NCT02223247 (2017).

125. US National Library of Medicine. ClinicalTrials.gov https://clinicaltrials.gov/ct2/show/NCT03179904 (2018).

126. US National Library of Medicine. ClinicalTrials.gov https://clinicaltrials.gov/ct2/show/NCT03032484 (2017).

127. US National Library of Medicine. ClinicalTrials.gov https://clinicaltrials.gov/ct2/show/NCT02891538 (2018). 
128. White, P. J. et al. The BCKDH kinase and phosphatase integrate BCAA and lipid metabolism via regulation of ATP-citrate lyase. Cell Metab. 27, 1281-1293 (2018).

129. Abdul-Wahed, A., Guilmeau, S. \& Postic, C. Sweet sixteenth for ChREBP: established roles and future goals. Cell Metab. 26, 324-341 (2017).

130. Yan, C. et al. The mTOR inhibitor rapamycin synergizes with a fatty acid synthase inhibitor to induce cytotoxicity in ER/HER2-positive breast cancer cells. PLOS ONE 9, e97697 (2014).

131. Takeuchi, K. \& Reue, K. Biochemistry, physiology, and genetics of GPAT, AGPAT, and lipin enzymes in triglyceride synthesis. Am. J. Physiol. Endocrinol. Metab. 296, E1195-E1209 (2009).

132. Quinn, W. J. III et al. mTORC1 stimulates phosphatidylcholine synthesis to promote triglyceride secretion J. Clin. Invest. 127, 4207-4215 (2017).

133. Kim, S. W., Kim, H. J., Chun, Y. J. \& Kim, M. Y. Ceramide produces apoptosis through induction of $\mathrm{p} 27$ (kip1) by protein phosphatase 2A-dependent Akt dephosphorylation in PC-3 prostate cancer cells. J. Toxicol. Environ. Health A 73, 1465-1476 (2010).

134. Morad, S. A. \& Cabot, M. C. Ceramide-orchestrated signalling in cancer cells. Nat. Rev. Cancer 13, 51-65 (2013).

135. Ader, I., Brizuela, L., Bouquerel, P., Malavaud, B. \& Cuvillier, O. Sphingosine kinase 1: a new modulator of hypoxia inducible factor 1alpha during hypoxia in human cancer cells. Cancer Res. 68, 8635-8642 (2008).

136. Bouquerel, P. et al. Essential role for SphK1/S1P signaling to regulate hypoxiainducible factor 2alpha expression and activity in cancer. Oncogenesis 5, e209 (2016).

137. Sano, O. et al. Using a biologically annotated library to analyze the anticancer mechanism of serine palmitoyl transferase (SPT) inhibitors. FEBS Open Bio 7, 495-503 (2017).

138. US National Library of Medicine. ClinicalTrials.gov https://clinicaltrials.gov/ct2/show/NCT03377179 (2018).

139. US National Library of Medicine. ClinicalTrials.gov https://clinicaltrials.gov/ct2/show/NCT02757326 (2018).

140. US National Library of Medicine. ClinicalTrials.gov https://clinicaltrials.gov/ct2/show/NCT02939807 (2018).

141. Xun, C. et al. Targeting sphingosine kinase 2 (SphK2) by ABC294640 inhibits colorectal cancer cell growth in vitro and in vivo. J. Exp. Clin. Cancer Res. 34, 94 (2015).

142. Mattaini, K. R., Sullivan, M. R. \& Vander Heiden, M. G. The importance of serine metabolism in cancer. J. Cell Biol. 214, 249-257 (2016).

143. Adams, C. M. Role of the transcription factor ATF4 in the anabolic actions of insulin and the anti-anabolic actions of glucocorticoids. J. Biol. Chem. 282, 16744-16753 (2007).

144. Gu, Y. et al. mTORC2 regulates amino acid metabolism in cancer by phosphorylation of the cystine-glutamate antiporter xCT. Mol. Cell 67, 128-138 (2017). 
145. Sahu, N. et al. Proline starvation induces unresolved ER stress and hinders mTORC1-dependent tumorigenesis. Cell Metab. 24, 753-761 (2016). This paper demonstrates the dependence on proline for mTORC1-dependent tumorigenesis in a subset of cancer cells.

146. $\mathrm{Gu}, \mathrm{X}$. et al. SAMTOR is an S-adenosylmethionine sensor for the mTORC1 pathway. Science 358, 813-818 (2017).

147. Castellano, B. M. et al. Lysosomal cholesterolactivates mTORC1 via an SLC38A9-Niemann-Pick C1 signaling complex. Science 335, 1306-1311 (2017).

148. Mayers, J. R. et al. Tissue of origin dictates BCAA metabolism in mutant Krasdriven cancers. Science 353, 1161-1165 (2016). This paper reports that cancerspecific changes in BCAA metabolism are driven by the tissue of origin rather than underlying mutations

149. Kankotia, S. \& Stacpoole, P. W. Dichloroacetate and cancer: new home for an orphan drug? Biochim. Biophys. Acta 1846, 617-629 (2014).

150. Skrott, Z. et al. Alcohol-abuse drug disulfiram targets cancer via p97 segregase adaptor NPL4. Nature 552, 194-199 (2017).

151. Howell, J. J. et al. Metformin inhibits hepatic mTORC1 signaling via dosedependent mechanisms involving AMPK and the TSC complex. Cell Metab. 25, 463-471 (2017).

152. Jara, J. A. \& Lopez-Munoz, R. Metformin and cancer: between the bioenergetic disturbances and the antifolate activity. Pharmacol. Res. 101, 102-108 (2015).

153. Pollak, M. N. Investigating metformin for cancer prevention and treatment: the end of the beginning. Cancer Discov. 2, 778-790 (2012).

154. Morales, D. R. \& Morris, A. D. Metformin in cancer treatment and prevention. Annu. Rev. Med. 66, 17-29 (2015).

155. Benjamin, D. et al. Syrosingopine sensitizes cancer cells to killing by metformin. Sci. Adv. 2, e1601756 (2016). This paper demonstrates synergistic effects of metformin and syrosingopine in several cancer models.

156. US National Library of Medicine. ClinicalTrials.gov https://clinicaltrials.gov/ct2/show/NCT01529593 (2018).

157. US National Library of Medicine. ClinicalTrials.gov https://clinicaltrials.gov/ct2/show/NCT02145559 (2018).

158. US National Library of Medicine. ClinicalTrials.gov https://clinicaltrials.gov/ct2/show/NCT02048384 (2017).

159. Kim, L. C., Cook, R. S. \& Chen, J. mTORC1 and mTORC2 in cancer and the tumor microenvironment. Oncogene 36, 2191-2201 (2017).

160. Sousa, C. M. et al. Pancreatic stellate cells support tumour metabolism through autophagic alanine secretion. Nature 536, 479-483 (2016). This paper shows that cancer-associated fibroblasts support tumour cells by secreting non-essential amino acids.

161. Duluc, C. et al. Pharmacological targeting of the protein synthesis mTOR/4EBP1 pathway in cancer- associated fibroblasts abrogates pancreatic tumour chemoresistance. EMBO Mol. Med. 7, 735-753 (2015).

162. Timosenko, E., Hadjinicolaou, A. V. \& Cerundolo, V. Modulation of cancer- 
specific immune responses by amino acid degrading enzymes. Immunotherapy 9, 83-97 (2017).

163. Pavlova, N. N. \& Thompson, C. B. The emerging hallmarks of cancer metabolism. Cell Metab. 23, 27-47 (2016).

164. Chang, C. H. et al. Metabolic competition in the tumor microenvironment is a driver of cancer progression. Cell 162, 1229-1241 (2015).

165. Bar-Peled, L., Schweitzer, L. D., Zoncu, R. \& Sabatini, D. M. Ragulator is a GEF for the rag GTPases that signal amino acid levels to mTORC1. Cell 150, 1196-1208 (2012).

166. Shen, K. et al. Architecture of the human GATOR1 and GATOR1-Rag GTPases complexes. Nature 556, 64-69 (2018).

167. Wolfson, R. L. et al. KICSTOR recruits GATOR1 to the lysosome and is necessary for nutrients to regulate mTORC1. Nature 543, 438-442 (2017).

168. Tsun, Z. Y. et al. The folliculin tumor suppressor is a GAP for the RagC/D GTPases that signal amino acid levels to mTORC1. Mol. Cell 52, 495-505 (2013).

169. US National Library of Medicine. ClinicalTrials.gov https://clinicaltrials.gov/ct2/show/NCT03163667 (2017).

Acknowledgements

D.M. acknowledges support from the German Research Foundation (DFG). M.N.H. acknowledges support from the European Research Council (ERC) (Mechanisms of Evasive Resistance in Cancer (MERiC)), SystemsX.CH, the Louis Jeantet Foundation and the Swiss National Science Foundation.

Author contributions

D.M. and S.P. researched data for the article, substantially contributed to the discussion of content, wrote the article and edited the manuscript before submission. M.N.H. substantially contributed to the discussion of content, wrote the article and edited the manuscript before submission. 
Fig. 1: Activation of mTOR signalling

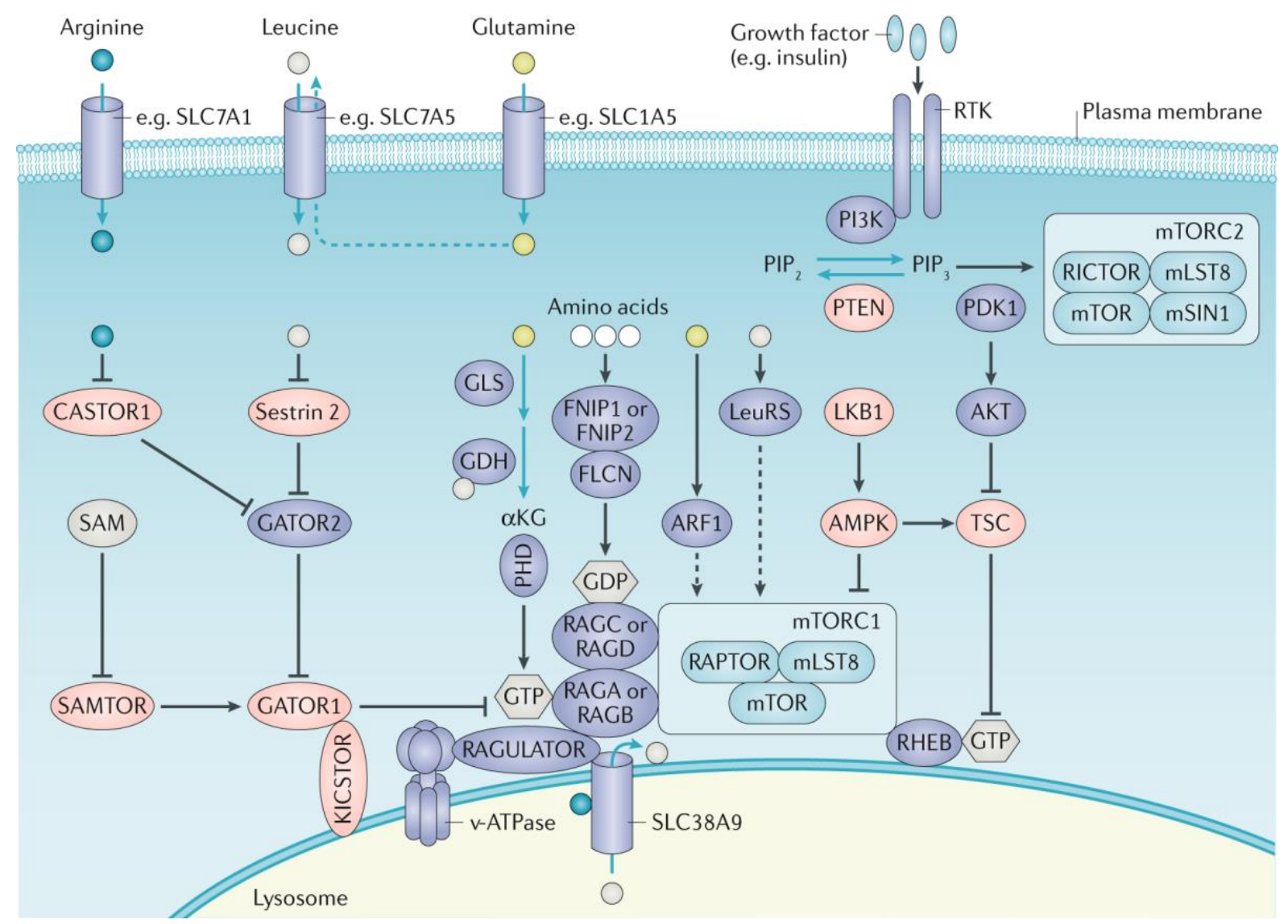

Growth factors (for example, insulin) bind to receptor tyrosine kinases (RTKs) to activate PI3K. PI3K converts phosphatidylinositol-4,5-bisphosphate (PIP2) to phosphatidylinositol-3,4,5-trisphosphate (PIP3), which is counteracted by PTEN. For activation of mTOR complex 2 (mTORC2), PIP3 binds mammalian stress-activated MAPK-interacting protein 1 (mSIN1). For activation of mTORC1, PIP3 recruits phosphoinositide-dependent kinase 1 (PDK1) to activate AKT. AKT inhibits the TSC complex, which acts as GTPase-activating protein (GAP) on the GTPase RHEB. GTP-bound RHEB binds and activates mTORC1. Energy stress activates liver kinase B1 (LKB1) and AMP-activated kinase (AMPK) to suppress mTORC1 via inhibition of regulatory-associated protein of mTOR (RAPTOR) and activation of the TSC complex ${ }^{107}$. Full activation of mTORC1 requires signalling induced by amino acids. SLC7A1, SLC7A5, SLC1A5 and other transporters import amino acids into the cell. Once in the cytoplasm, amino acids can induce activation of RAS-related GTP-binding proteins (RAGs), which mediate lysosomal translocation of mTORC1. RAGULATOR binds the RAGs at the lysosome and acts as a guanine nucleotide exchange factor towards RAGA and/or RAGB ${ }^{165}$. GAP activity towards RAGS 1 (GATOR1) inactivates RAGA and/or RAGB ${ }^{166}$. KICSTOR recruits and binds GATOR1 (ref. ${ }^{167}$ ). GATOR2 is required for mTORC1 activation and was suggested to bind and inhibit GATOR1. Leucine can promote mTORC1 activation via binding to sestrin 2 and/or sensing by LeuRS. Arginine binds to CASTOR1. Both sestrin 2 and CASTOR1 release inhibition of GATOR2 $\left(\right.$ ref $^{10}{ }^{10}$ ). Glutamine activates mTORC1 in a RAG-dependent manner via glutaminolysis and in a RAGindependent manner via the small GTPase ARF1. The lysosomal transporter SLC38A9 exports essential amino acids into the cytosol in an arginine-stimulated fashion ${ }^{11}$. Amino acids signal through folliculin (FLCN) and FLCN-interacting protein 1 (FNIP1) or FNIP2, which act as GAPs for RAGC and/or RAGD ${ }^{168}$. S-adenosyl-methionine (SAM) prevents SAMTOR binding to GATOR1 and KICSTOR $^{146}$. Light red-coloured molecules indicate negative and purple coloured molecules indicate positive regulators of mTOR signalling. Turquoise arrows indicate metabolite flux, while black arrows indicate a signalling cascade. Dashed lines indicate indirect actions or that it is unknown whether additional steps are required. $\alpha \mathrm{KG}, \alpha$-ketoglutarate; GDH, glutamate dehydrogenase; GLS, glutaminase; mLST8, mammalian lethal with sec-13 protein 8; PHD, prolyl hydroxylase. 


\section{$\underset{\text { a ciutamine metabolism }}{\text { Fig. 2: mTriven reprogramming of amino acid metabolism }}$}
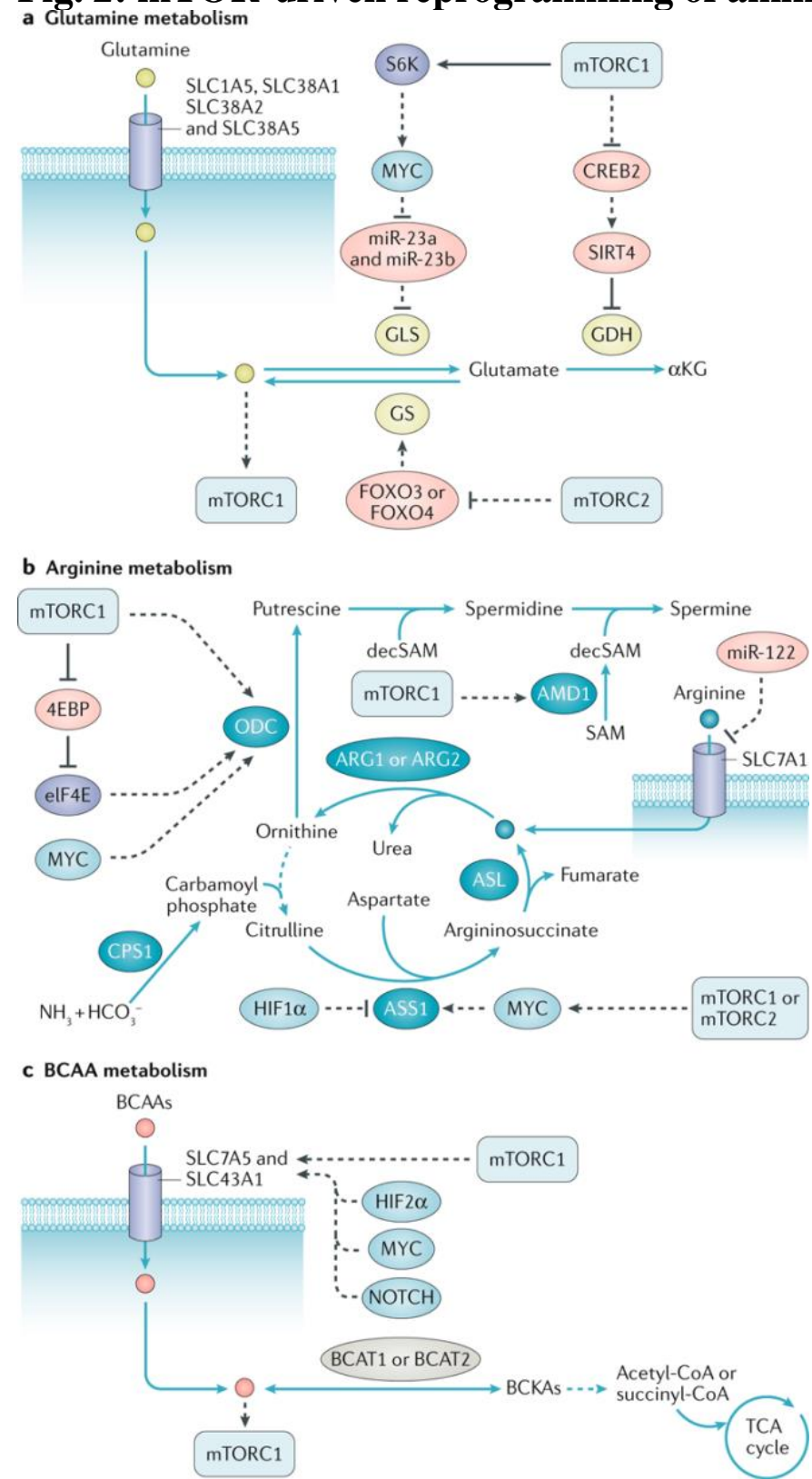

a | Glutamine metabolism. SLC1A5, SLC38A1, SLC38A2 and SLC38A5 mediate glutamine uptake. Glutamine, which contributes to activation of mTOR complex 1 (mTORC1), is converted to glutamate and $\alpha$-ketoglutarate ( $\alpha \mathrm{KG}$ ) by glutaminase (GLS) and glutamate dehydrogenase (GDH). mTORC1 phosphorylates and thereby activates ribosomal protein S6 kinase (S6K). S6K promotes MYC translation, which derepresses GLS by inhibiting miR-23a and miR-23b. mTORC1 also induces proteasome-dependent degradation of cAMP-responsive element binding 2 (CREB2), a transcription factor that induces sirtuin 4 (SIRT4) expression. SIRT4 inhibits GDH by ADP ribosylation. Glutamine synthetase (GS) converts glutamate to glutamine. GS expression is promoted by FOXO3 and/or FOXO4, which are potentially under negative control of mTORC2. b $\mid$ Arginine metabolism. SLC7A1 mediates arginine uptake. Carbamoyl phosphate synthetase 1 (CPS1) produces carbamoyl phosphate, which condenses with ornithine to form citrulline. Argininosuccinate synthetase 1 (ASS1) ligates citrulline and aspartate to form argininosuccinate. Argininosuccinate is cleaved to arginine and fumarate by argininosuccinate lyase (ASL). Arginine is cleaved to urea and ornithine by cytosolic arginase 1 (ARG1) or mitochondrial arginase 2 (ARG2). During polyamine synthesis, ornithine is decarboxylated by ornithine decarboxylase (ODC) to form putrescine. Putrescine serves as the precursor for the formation of the polyamines spermidine and spermine. Adenosylmethionine decarboxylase 1 (AMD1) decarboxylates S-adenosyl-methionine (SAM) to decarboxylated SAM 
(decSAM), which is required for polyamine synthesis. mTORC1 and/or mTORC2 promote reactions as shown. $\mathbf{c}$ Branched chain amino acid (BCAA) metabolism. SLC7A5 and SLC43A1 mediate BCAA uptake. Branched chain aminotransferase 1 (BCAT1) or BCAT2 mediate interconversion of BCAAs and branched chain ketoacids (BCKAs). BCKAs are decarboxylated by BCKA dehydrogenase (BCKD), and further catabolism results in formation of acetyl-CoA and succinyl-CoA, which enter the tricarboxylic acid (TCA) cycle. mTORC1 may promote BCAA uptake. Turquoise arrows indicate metabolite flux, while black arrows indicate a signalling cascade. Dashed lines indicate indirect actions or that it is unknown whether additional steps are required. 4EBP, eIF4E binding protein; HCO3-, bicarbonate; HIF, hypoxia-inducible factor; NH3, ammonia. 
Fig. 3: mTOR controls glucose metabolism in cancer

Glucose

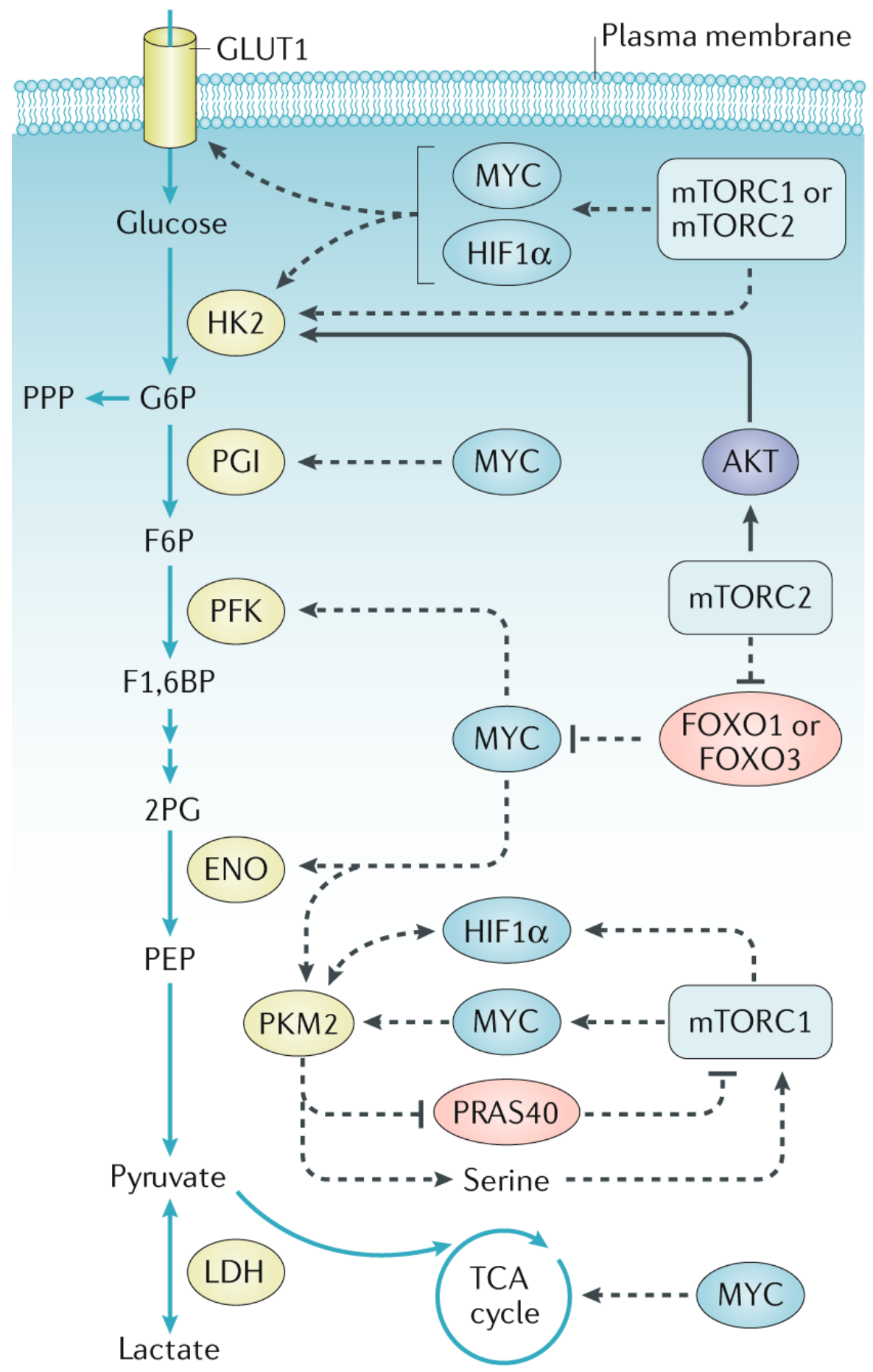

Glucose transporter 1 (GLUT1) mediates glucose uptake. Glucose is phosphorylated by hexokinase 2 (HK2) to form glucose-6-phosphate (G6P), which can be diverted into the pentose phosphate pathway (PPP). Phosphoglucoisomerase (PGI) isomerizes G6P to fructose-6-phosphate (F6P), which is further phosphorylated by phosphofructokinase (PFK) to form fructose-1,6-bisphosphate (F1,6BP). Multiple enzymatic steps lead to the formation of 2-phosphoglycerate (2PG), which is converted to phosphoenolpyruvate (PEP) by enolase (ENO). In the last step of glycolysis, PEP is converted to pyruvate by pyruvate kinase muscle isoform 2 (PKM2). Lactate dehydrogenase (LDH) interconverts pyruvate and lactate. mTOR complex 1 (mTORC1) and/or mTORC2 promote glycolysis by direct mechanisms or indirectly by induction of the transcription factors MYC and/or hypoxia-inducible factor $1 \alpha(\mathrm{HIF} 1 \alpha)$. PKM2 also acts back on mTORC1 by promoting serine biosynthesis and by inhibiting proline-rich AKT substrate 1 (PRAS40), a negative regulator of mTORC1. Turquoise arrows indicate metabolite flux, while black arrows indicate a signalling cascade. Dashed lines indicate indirect actions or that it is unknown whether additional steps are required. TCA, tricarboxylic acid. 
Fig. 4: Rewiring of nucleotide metabolism by mTOR

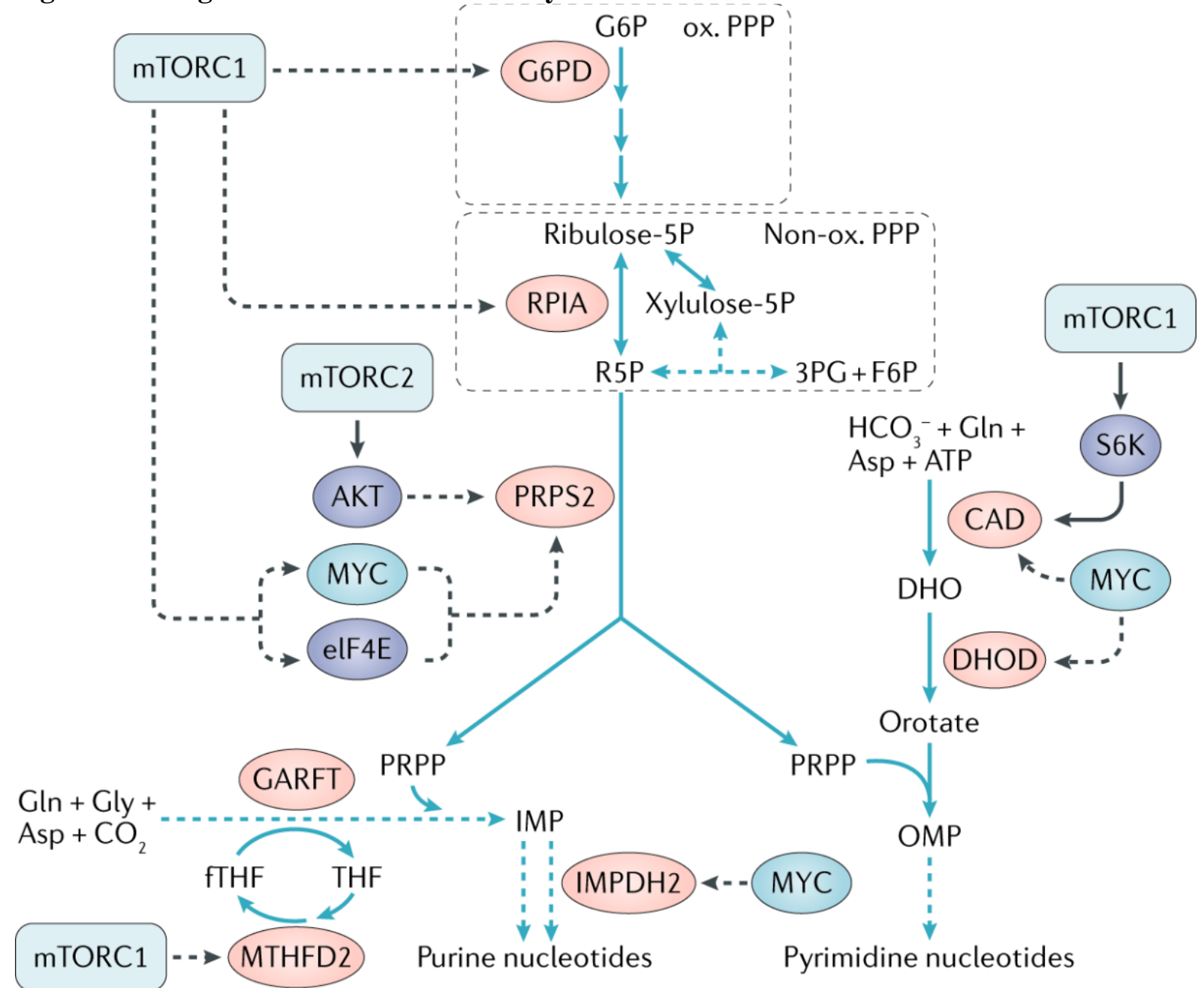

Glucose-6-phosphate (G6P) derived from glycolysis fuels the pentose phosphate pathway (PPP) to generate ribose-5-phosphate (R5P) for nucleotide synthesis. The PPP is divided into an oxidative and a non-oxidative branch (ox. PPP and non-ox. PPP, respectively), which involve enzymes such as G6P dehydrogenase (G6PD) and R5P isomerase A (RPIA), both of which are stimulated by mTOR complex 1 (mTORC1). Phosphoribosyl pyrophosphate synthase 2 (PRPS2) ultimately generates ribose-5phosphoribosyl-1-pyrophosphate (PRPP) from R5P and is induced by mTORC1 and mTORC2 through independent mechanisms, as shown. Purine synthesis requires glutamine, glycine, aspartate, formyltetrahydrofolate (fTHF) and CO2. The formation of fTHF is promoted by mTORC1 through methylenetetrahydrofolate dehydrogenase 2 (MTHFD2). The purine ring is assembled directly on PRPP. Multiple enzymes, including glycinamide ribonucleotide formyltransferase (GARFT), lead to the formation of inosine monophosphate (IMP). IMP is further converted to different forms of purine nucleotides. The formation of some purine nucleotides requires IMP dehydrogenase 2 (IMPDH2). Assembly of the pyrimidine ring requires glutamine, aspartate, ATP and bicarbonate ( $\mathrm{HCO}^{-}{ }^{-}$). Initial steps are carried out by carbamoyl phosphate synthetase 2, aspartate transcarbamylase and dihydroorotase (CAD) to form dihydroorotate (DHO). mTORC1, via ribosomal protein S6 kinase (S6K), promotes CAD activity. Orotate is combined with PRPP to generate orotidine monophosphate (OMP). OMP is the precursor for all pyrimidine nucleotides. Turquoise arrows indicate metabolite flux, while black arrows indicate a signalling cascade. Dashed lines indicate indirect actions or that it is unknown whether additional steps are required. 3PG, 3-phosphoglycerate; DHOD, DHO dehydrogenase; F6P, fructose-6-phosphate. 
Fig. 5: Regulation of fatty acid and lipid metabolism

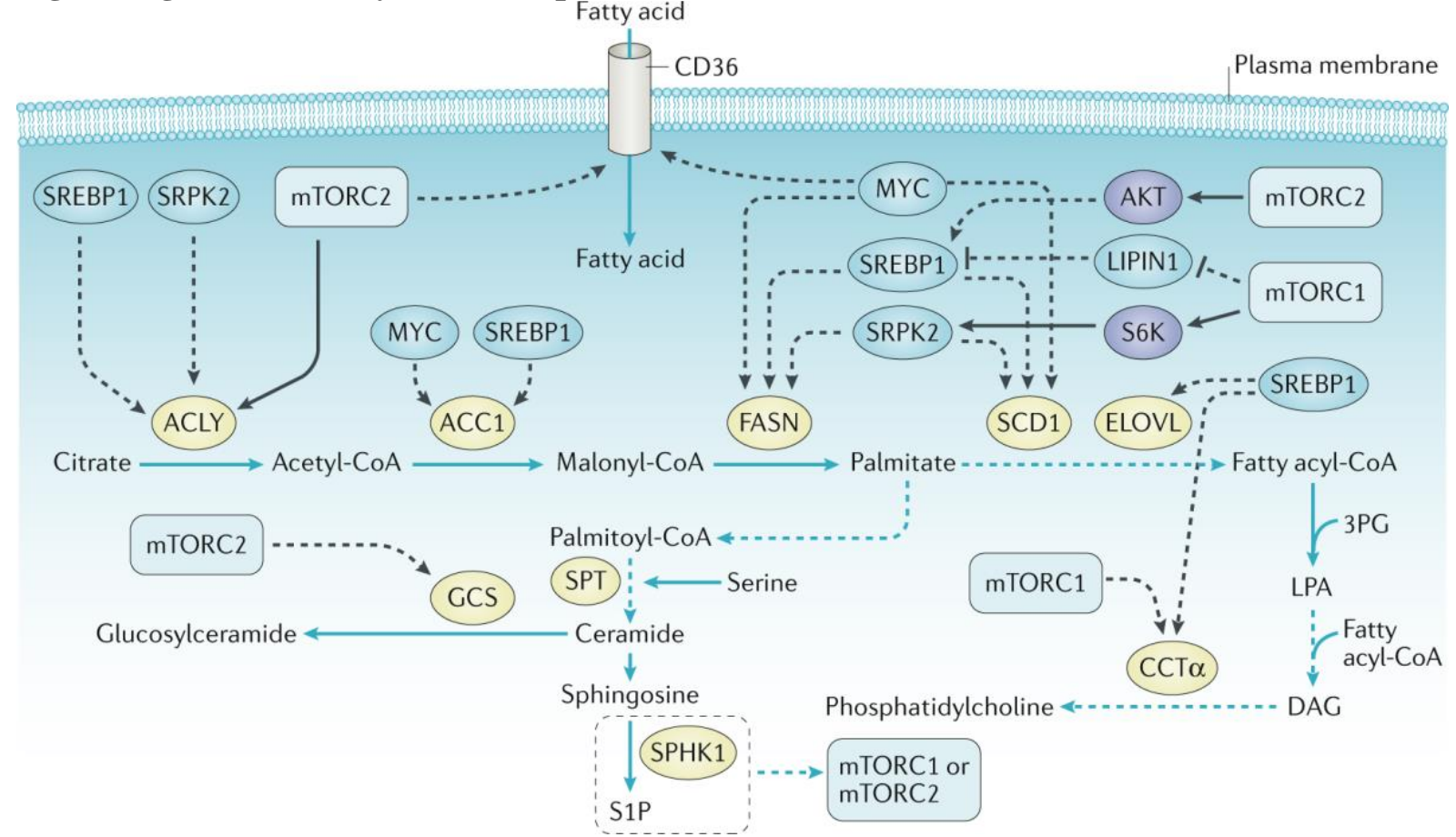

CD36 mediates fatty acid uptake. For de novo fatty acid synthesis, ATP citrate lyase (ACLY) converts citrate derived from the tricarboxylic acid cycle (TCA) cycle, for example, to acetyl-CoA. Acetyl-CoA carboxylase 1 (ACC1) catalyses the carboxylation of acetyl-CoA to malonyl-CoA. Fatty acid synthase (FASN) synthesizes the fatty acid palmitate by condensing acetyl-CoA groups with malonyl-CoA. Elongation and desaturation reactions starting from palmitate result in different fatty acid species. Lysophosphatidic acid (LPA) is synthesized from fatty acyl-CoA and 3-phosphoglycerate (3PG). Addition of another fatty acyl-CoA molecule leads to the formation of diacylglycerol (DAG). DAG can be converted into different phosphoglycerides, for example, phosphatidylcholine. mTOR complex 1 (mTORC1) promotes phosphatidylcholine synthesis by stimulating phosphocholine cytidylyltransferase- $\alpha(\mathrm{CCT} \alpha)$. In de novo sphingolipid synthesis, palmitoyl-CoA is converted into ceramide through multiple enzymatic reactions involving serine palmitoyltransferase (SPT). Ceramide serves as a precursor for at least four different sphingolipid species (sphingomyelin, sphingosine, ceramide-1-phosphate and glucosylceramide). Glucosylceramide synthase (GCS) converts ceramide to glucosylceramide, which can be promoted by mTORC2. In addition, ceramide is sequentially converted to sphingosine and phosphorylated by sphingosine kinase 1 (SPHK1) to form sphingosine-1phosphate (S1P). S1P can promote mTORC1 and mTORC2 signalling. Turquoise arrows indicate metabolite flux, while black arrows indicate a signalling cascade. Dashed lines indicate indirect actions or that it is unknown whether additional steps are required. ELOVL, elongation of very long chain fatty acids; S6K, ribosomal protein S6 kinase; SREBP1, sterol regulatory element binding protein 1; SRPK2, serine/arginine-rich protein kinase 2. 
Table 1 Ongoing clinical trials of drugs targeting cancer metabolism

\begin{tabular}{|c|c|c|c|}
\hline Target molecule or pathway & Drug name & Cancer type & Refs \\
\hline \multicolumn{4}{|l|}{ Amino acid metabolism } \\
\hline \multirow[t]{3}{*}{ GLS } & CB-839 & Breast cancer & 32 \\
\hline & & $\begin{array}{l}\text { Breast cancer, RCC, } \\
\text { melanoma, mesothelioma, } \\
\text { etc. }\end{array}$ & 33 \\
\hline & CB-839 + everolimus ${ }^{a}$ & $\mathrm{RCC}$ & 169 \\
\hline \multirow[t]{4}{*}{ Arginine } & rhARG1 & Acute myeloid leukaemia & 44 \\
\hline & & $\begin{array}{l}\text { HCC, RCC, melanomaand } \\
\text { prostate adenocarcinoma }\end{array}$ & 45 \\
\hline & ADI-PEG20 & Prostate cancer and NSCLC & 46 \\
\hline & & Advanced solid tumour & 47 \\
\hline \multicolumn{4}{|l|}{ Glucose metabolism } \\
\hline MCT1 & AZD-3965 & $\begin{array}{l}\text { Prostate cancer, gastric cancer } \\
\text { and diffuse large B cell } \\
\text { lymphoma }\end{array}$ & 93 \\
\hline \multicolumn{4}{|l|}{ Fatty acid metabolism } \\
\hline \multirow[t]{5}{*}{ FASN } & TVB-2640 & Solid malignant tumour & 124 \\
\hline & & Colon cancer & 123 \\
\hline & & Breast cancer & 125 \\
\hline & & Astrocytoma & 126 \\
\hline & EGCG & Colorectal cancer & 127 \\
\hline \multirow[t]{3}{*}{ SPHK2 } & ABC294640 & Advanced cholangiocarcinoma & 138 \\
\hline & & Multiple myeloma & 139 \\
\hline & & Advanced HCC & 140 \\
\hline \multicolumn{4}{|l|}{ Glucose and fatty acidmetabolism } \\
\hline \multirow[t]{3}{*}{ Glycolysisand fattyacidsynthesis } & Metformin + temsirolimus $^{a}$ & Advanced cancers & 156 \\
\hline & Metformin + rapamycin & $\begin{array}{l}\text { Breastneoplasms,lung neoplasms, } \\
\text { liver cancer,lymphomaandkidney } \\
\text { cancer }\end{array}$ & 157 \\
\hline & & Pancreatic cancer & 158 \\
\hline
\end{tabular}

ADI-PEG20, pegylated arginine deiminase; EGCG, epigallocatechin gallate; FASN, fatty acid synthase; GLS, glutaminase; HCC, hepatocellular carcinoma; MCT1, monocarboxylate transporter 1; NSCLC, non-small-cell lung cancer; RCC, renal cell carcinoma; rhARG1, recombinant human cytosolic arginase 1; SPHK2, sphingosine kinase 2. aIndicates an mTOR complex 1 (mTORC1) inhibitor. 


\section{Box $1 \mathrm{mTOR}$ signalling and metabolic flux in the tumour microenvironment}

Recent studies have revealed extensive signalling and metabolic flux between tumour cells and nearby non-tumour cells, such as fibroblasts or T cells (see the figure).

\section{Fibroblasts}

In pancreatic ductal adenocarcinoma (PDAC), cancer-associated fibroblasts (CAFs) support tumour cell growth by secreting non-essential amino acids, in particular, alanine. PDAC cells use alanine to fuel the tricarboxylic acid (TCA) cycle and to synthesize lipids. This alanine flux within the tumour microenvironment (TME) enables the tumour cells to divert glucose for the synthesis of non-essential amino acids, such as serine and glycine, which are required for nucleotide synthesis ${ }^{160}$. Whether mTOR signalling is involved in this metabolic modulation is not known; however, CAFs in PDAC possess high mTOR activity ${ }^{161}$. If it is, the use of an mTOR inhibitor might be beneficial, as it would inhibit CAFs and thereby interrupt metabolic flux between CAFs and tumour cells.

\section{T cells}

Amino acid flux. Cancer cells counter immune cells by depleting the TME of specific amino acids. Secretion of cytosolic arginase 1 (ARG1) or mitochondrial arginase 2 (ARG2) leads to reduced levels of arginine and inhibition of $\mathrm{T}$ cells in the $\mathrm{TME}^{162}$. Overexpression of indoleamine-2,3-dioxygenase 1 (IDO1) and tryptophan-2,3-dioxygenase 2 (TDO2) leads to the conversion of the essential amino acid tryptophan into kynurenine. While tryptophan deprivation triggers apoptosis of T cells in the TME, secreted kynurenine promotes a regulatory $\mathrm{T}$ cell phenotype, which further inhibits immune responses ${ }^{163}$.

Glycolytic flux. In a mouse sarcoma model, tumour cells outcompete T cells for glucose. This leads to sustained mTOR signalling and glycolysis in the tumour cells and, as a consequence, tumour progression. Conversely, decreased mTOR signalling, glycolysis and chemokine secretion in the T cells prevent the T cells from checking tumour progression ${ }^{164}$. Furthermore, as a result of increased glycolysis, tumour cells often secrete lactate via monocarboxylate transporter 4 (MCT4), which affects the TME by promoting immunosuppression and angiogenesis ${ }^{163}$.

\section{Metabolic symbiosis via lactate}

mTOR-dependent metabolic symbiosis between cancer cells, in which cancer cells can take up lactate from the TME via MCT1 and use it for metabolism, also occurs, as further described in the main text $^{91,92}$. 


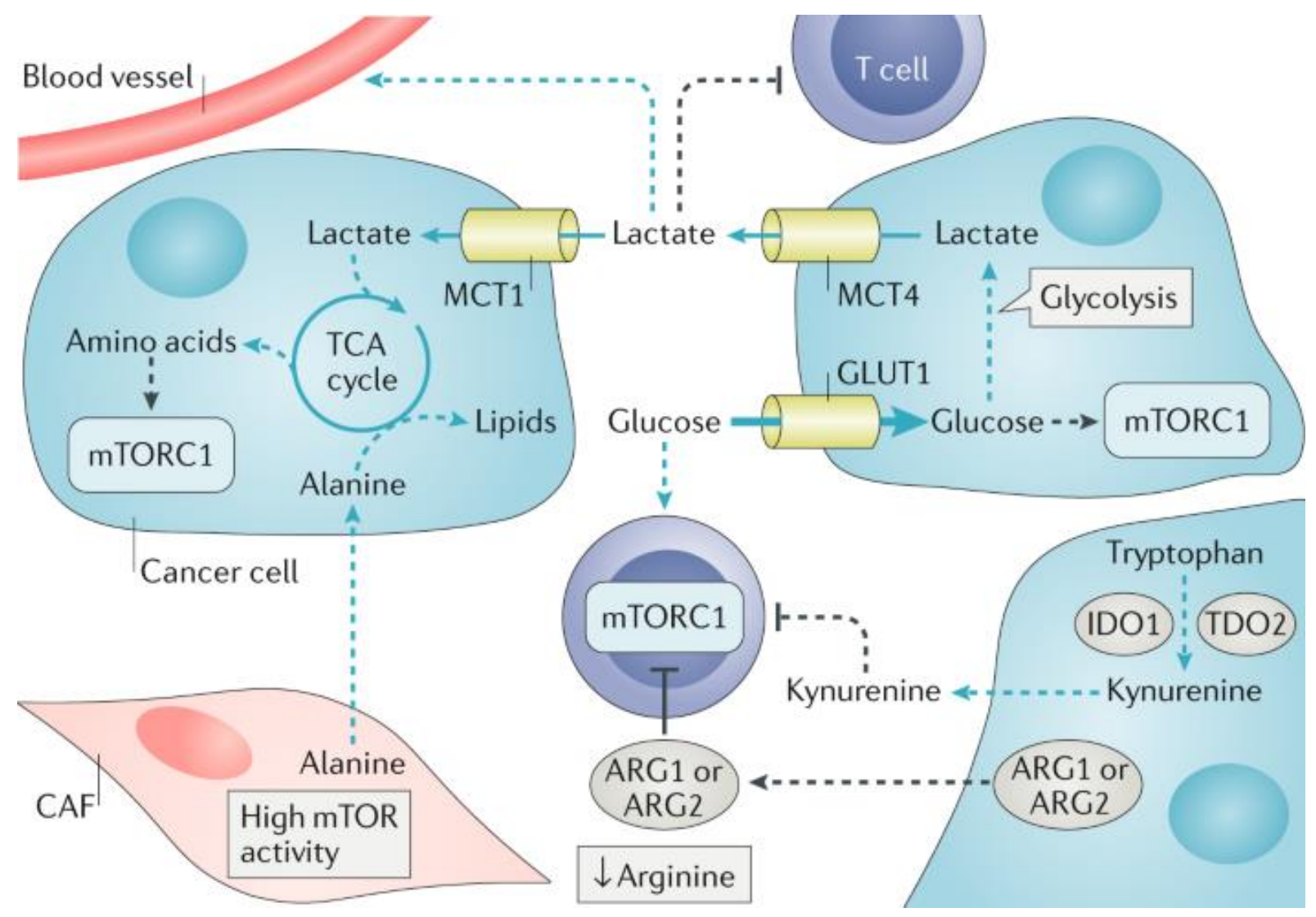

